KIAS-P09008

SU-ITP-09/10

\title{
Holographic Deuteron and Nucleon-Nucleon Potential
}

\author{
Youngman Kim^${ }^{\bullet}$, Sangmin Lee ${ }^{\diamond}$ and Piljin $\mathrm{Yi}^{\star}$ \\ - School of Physics, Korea Institute for Advanced Study, Seoul 130-722, Korea \\ ${ }^{\circ}$ Asia Pacific Center for Theoretical Physics, Pohang, Gyeongbuk 790-784, Korea \\ $\diamond$ Department of Physics, Seoul National University, Seoul 151-747, Korea
}

\begin{abstract}
We compute the potential between a pair of nucleons in the D4-D8 holographic QCD. In the large 't Hooft coupling limit, $\lambda \gg 1$, the hadronic size of the baryon is small $\sim 1 / \sqrt{\lambda} M_{K K}$, and their interaction with mesons are well approximated by a set of dimension four and five operators. The nucleon-nucleon potential emerges from one-boson exchange picture involving massless pseudo-scalars and an infinite tower of spin one mesons. We find in particular that $\rho$ meson exchanges are dominated by a dimension five derivative coupling of tensor type, whereas for $\omega$ mesons and axial mesons, such tensor couplings are completely absent. The potential is universally repulsive $\sim 1 / r^{2}$ at short distance, and has the usual long-distance attractive behavior $\sim-1 / r^{3}$ along a isosinglet and spin triplet channel. Both the large $N_{c}$ form and the finite $N_{c}$ form are given. In the former, a shallow classical minimum of depth $\sim 0.1 M_{K K} N_{c} / \lambda$ forms at around $r M_{K K} \simeq 5.5$.
\end{abstract}




\section{Contents}

1 Nucleon-Nucleon Potential from Holography 1

2 A D4-D8 Holographic QCD 4

3 Holographic Baryons

4 Nucleon-Meson Interactions from Holography

4.1 General Formulation . . . . . . . . . . . . . . . . . 10

4.2 Structure of the Cubic Couplings . . . . . . . . . . . . . . . . . . 12

4.3 Scaling of the Cubic Couplings . . . . . . . . . . . . . . 13

4.4 Pseudo-scalar Mesons: $\pi$ and $\eta^{\prime} \ldots \ldots \ldots \ldots$. . . . . . . . . . . . . . . . . . . . . . . . .

4.5 Vector Mesons: $\rho$ and $\omega \ldots \ldots \ldots \ldots$. . . . . . . . . . . . . . . . . . . . . . . . . . .

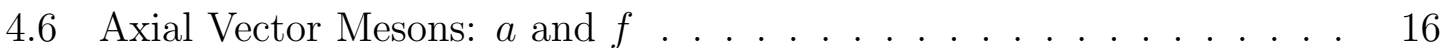

5 Large $N_{c}$ Nucleon-Nucleon Potential

5.1 Holographic Nucleon-Nucleon Potential . . . . . . . . . . . . . . . 18

5.2 Behavior at $r \sim 1 / M_{K K} \gg 1 / \sqrt{\lambda} M_{K K} \ldots \ldots \ldots . \ldots \ldots$

5.3 Coulomb Repulsion at Short Distance and Finite $\lambda$ Corrections to the Large $N_{c}$ Potential . . . . . . . . . . . . . . . . . . . 21

6 Nucleon-Nucleon Potential for Realistic $\lambda$ and $N_{c} \quad 25$

7 Holographic Deuteron: Large $N_{c}$ Results 29

8 Concluding Remarks

\section{Nucleon-Nucleon Potential from Holography}

The main goal of this paper is to extract the interaction between a pair of nucleons in string theoretical framework of holography, and consider possible bound state. We will exclusively work with the D4-D8 model [1], which involves a large number of colors $N_{c}$, large 't Hooft coupling $\lambda$, and quenching of fermions. Given many approximations, the result should be approached with much caution, yet we have seen often that such holographic approaches generating realistic numbers. Ref. [2, 3, for instance, gave detailed predictions on glueball spectrum of pure QCD, some of which were successfully compared to lattice simulation.

The D4-D8 model has been particularly successful in encoding the spin 1 meson sector coupled with pseudo-Goldstone bosons, and equally successful in describing baryons and the interaction between these two sectors [1, 4, 5, 6]. A natural extension 
of the model would be a study of the nucleon-nucleon potential. The holographic baryon in the D4-D8 model is similar to the Skyrmion of chiral perturbation theory. In fact, one can view the holographic baryon as a direct uplift of the Skyrmion in the holographic sense, which has a simple interpretation as instanton solitons with certain Coulombic electric hair. The size of the soliton is known to be $\simeq 9.6 / \sqrt{\lambda} M_{K K}$ [4, [5], where $M_{K K}$ is some natural unit in the D4-D8 model, comparable to the lightest vector meson mass.

Given this, one can approach the problem of nucleon potential in two different manners. The first, which seems conceptually most natural, is to find a "suitable" family of two-soliton trial configuration, emulating a pair of baryons (nucleons) taken apart from each other, and evaluate the resulting energy. After subtracting twice the mass of the baryon (nucleon), this would give us a potential. However, this is much easier said than done. The main problem with approaches like this is that finding a "suitable" configuration is all but impossible for complicated solitons like this. As we will see later, the baryon-baryon interaction scales as $N_{c} / \lambda$ whereas their masses scale as $N_{c} \lambda$, so the interaction accounts for a very small part of the two body energy. Unless our trial configuration is extremely fine-tuned, energy cost due to any slight error could easily overrun the interaction energy, resulting in a nonsensical answer \#1

Sometimes, however, the interaction energy grows substantially and the physical mechanism responsible for the interaction is easy to single out. For our solitonic baryon this happens when the two baryons approach each other to a distance comparable to their individual soliton size. The leading contribution comes from the fact that each unit soliton comes with Coulombic hair. Each baryon has $N_{c}$ unit of charges with the squared electric coupling $\sim 1 / N_{c} \lambda$, so one finds a repulsive core interaction of type

$$
V_{\text {core }} \sim \frac{N_{c}}{\lambda} \frac{1}{M_{K K} r^{2}}
$$

with $r^{2} \simeq \lambda / M_{K K}^{2}$ or less. Here $r$ denotes the mutual separation of the two baryons and the $1 / r^{2}$ behavior originates from the fact that the soliton lives in approximate $R^{4+1}$.

Nevertheless, this short distance behavior gives little insight to some common questions like how bound states, such as deuteron and other nuclei, form. In fact, it is not clear whether there is a realistic regime where the precise functional behavior of the repulsive core such as this can be measured, since, at short distances, the asymptotic freedom takes over and nucleons begin to see each other as collection of partons. Also the D4-D8 model, or any other holographic model based on gravity only, becomes somewhat dubious in the high energy regime well beyond $M_{K K}$ because of many non-QCD modes that begin to populate at $M_{K K}$ and higher.

\footnotetext{
${ }^{\# 1}$ In fact, approaches of this kind have been already tried for Skyrmions with mixed results [7, 8].
} 
If one is interested in longer distances where the interaction is potentially attractive and where, more to the point, the validity of the present approximation can meet real QCD, we must consider a different approach\#2 When the inter-baryon distance is larger that the sizes of the baryons, the baryons can be taken to be a point-like object. In such situation, the interactions can be all ascribed to exchange of light particles, namely mesons. Instead of trying to understand intricate structure of multi-solitons, one merely computes Feynman diagrams using (cubic) interaction vertices involving baryon currents and light mesons, such as pions $\pi$ and rho mesons $\rho$. Typical vertices that enter this computation are

$$
\overline{\mathcal{N}}(x) \Gamma \phi(x) \mathcal{N}(x)
$$

for the (pseudo-)scalar mesons, and

$$
\overline{\mathcal{N}}(x) \gamma_{\mu} \Gamma v^{\mu}(x) \mathcal{N}(x), \quad \overline{\mathcal{N}}(x) \gamma^{\mu \nu} \Gamma \partial_{\mu} v_{\nu}(x) \mathcal{N}(x)
$$

for (axial-)vector mesons, with $\Gamma=1$ or $\gamma^{5}$. From the D4-D8 holographic QCD, coupling constants for these vertices are all precisely derivable, at least in the large $\lambda$ and large $N_{c}$ limit. Then the problem of finding nucleon-nucleon potential becomes a matter of computing summing up tree-level Feynman diagrams due to various meson exchanges [9, 10, 11] \#3

Fortunately, the basic framework for the relevant meson-nucleon interaction has been worked out in great detail [4], where all nucleon-meson couplings can be derived very precisely. Some of the leading interaction strengths, such as the leading axial coupling to pions $g_{A}$ and vector meson couplings $g_{\rho \mathcal{N N}}$ and $g_{\omega \mathcal{N} \mathcal{N}}$, have been computed and successfully compared to experimental data. In this note, we will take this holographic formulation of nucleon-meson interaction and compute the nucleonnucleon potential from exchange of mesons. For distances $r \gg \sqrt{\lambda} / M_{K K}$, we find the leading potential of type

$$
V_{\text {exchange }} \sim \frac{N_{c}}{\lambda}(\cdots),
$$

where the ellipsis contains terms of order $1 / r$ through $1 / M_{K K}^{2} r^{3}$ possibly with exponential damping factor due to vector meson masses and also with various spin/isospin factors. Subleading contributions start at $1 / N_{c} \lambda$, but could be relevant in the real QCD regime of $N_{c}=3$.

However, this is not to say that the underlying mechanism for the interaction energy is different from what one would have obtained from the soliton approach if the latter were possible at all. Rather, the tree diagrams involving mesons and

\footnotetext{
\#2 The reverse is also true. One should not be tempted to use the second approach for the shortdistance interaction, since, with much more energy involved, the deformation of individual solitons is inevitable.

${ }^{\text {\#3}}$ See Ref. 12] for a comprehensive review in the conventional approach to QCD.
} 
baryons keep track of the classical effect on one baryon by another far away, and vice versa, and extract the classical interaction energy automatically. This is possible because the baryons are really solitons made out of these mesons, to begin with.

In section 2 and 3, we give a bare-bone review of D4-D8 holographic QCD and the solitonic baryon thereof. In section 4 , we derive relevant meson-nucleon couplings with emphasis on how they scale with $\lambda$ and $N_{c}$. In doing so, we will learn that certain derivative coupling of vector mesons, sometimes referred to as the tensor coupling, can be dominant over the usual minimal type couplings. We extract the values of these couplings in the large $N_{c}$ limit.

Section 5 and 6 summarize the resulting nucleon-nucleon potential, after suitable truncation by mass, for large $N_{c}$ and finite $N_{c}$ respectively. It will become clear that the leading contributions come from exchange of $\pi, \omega$, and axial-vector mesons via the minimal couplings, and from exchange of $\rho$ mesons via the tensor coupling. $\omega$ meson exchange is universally repulsive and represents a remnant of the core repulsion we mentioned earlier, while $\rho$ exchange off-set much of the pion exchange. For very short distances $\sim / \sqrt{\lambda} M_{K K}$, where the current approach become unreliable due to backreaction of the individual solitons, the potential turns universally repulsive as $\sim 1 / r^{2}$ as noted above.

Section 7 gives a simplistic view of deuteron emerging from the large $N_{c}$ form of the potential, and we close with a summary in section 8 .

\section{A D4-D8 Holographic QCD}

One starts with a stack of D4 branes which is compactified on a thermal circle [13, where one requires anti-periodic boundary condition on all fermions along the circle. The purpose of having a spatial "thermal" circle is to give mass to the fermionic superpartners and thus break supersymmetry. By putting $N_{c} \mathrm{D} 4$ branes on a thermal circle, we obtains pure $U\left(N_{c}\right)$ Yang-Mills theory in the remaining noncompact $3+1$ dimensions. We are interested in large $N_{c}$ limit, so the $U(1)$ part can be safely ignored, and we may pretend that we are studying $S U\left(N_{c}\right)$ theory instead. One then extrapolates the AdS/CFT [14 to this non-conformal case, which states that, instead of studying strongly coupled large $N_{c}$ Yang-Mills theory, one may look at its dual closed string theory. The correct dual geometry is known to be 15

$$
d s^{2}=\left(\frac{U}{R}\right)^{3 / 2}\left(\eta_{\mu \nu} d x^{\mu} d x^{\nu}+f(U) d \tau^{2}\right)+\left(\frac{R}{U}\right)^{3 / 2}\left(\frac{d U^{2}}{f(U)}+U^{2} d \Omega_{4}^{2}\right)
$$

with $R^{3}=\pi g_{s} N_{c} l_{s}^{3}$ and $f(U)=1-U_{K K}^{3} / U^{3}$. The topology of the spacetime is $R^{3+1} \times D \times S^{4}$, with the coordinate $\tau$ labeling the azimuthal angle of the disk $D$, with $\tau=\tau+\delta \tau$ and $\delta \tau=4 \pi R^{3 / 2} /\left(3 U_{K K}^{1 / 2}\right)$. The circle parameterized by $\tau$ is the 
thermal circle. The dilaton is

$$
e^{-\Phi}=\frac{1}{g_{s}}\left(\frac{R}{U}\right)^{3 / 4}
$$

while the antisymmetric Ramond-Ramond background field $C_{3}$ is such that $d C_{3}$ carries $N_{c}$ unit of flux along $S^{4}$.

To add mesons, one introduces $N_{F}$ D 8 branes, which share the coordinates $x^{\mu}$ with the above D4 branes [1] and are transverse to the thermal circle $\tau$. If we had not traded off the $N_{c}$ D4 branes in favor of the dual gravity theory, this would have allowed massless quarks as open strings ending on both the D4 and the D8 branes. As the D4's are replaced by the dual geometry, however, the 4-8 open strings have to be paired up into 8-8 open strings, the lightest of which belongs to a $U\left(N_{F}\right)$ gauge field, and these are naturally identified as bi-quark mesons. The $U\left(N_{F}\right)$ gauge theory on D8 branes has the action

$$
-\frac{4 \pi^{2} l_{s}^{4} \mu_{8}}{8} \int \sqrt{-h_{8+1}} e^{-\Phi} \operatorname{tr} \mathcal{F}^{2}+\mu_{8} \int C_{3} \wedge \operatorname{Tr} e^{2 \pi \alpha^{\prime} \mathcal{F}}
$$

where the contraction is via the induced metric of D8 and $\mu_{p}=2 \pi /\left(2 \pi l_{s}\right)^{p+1}$ with $l_{s}^{2}=\alpha^{\prime}$. The induced metric on the D8 brane is

$$
h_{8+1}=\frac{U^{3 / 2}(w)}{R^{3 / 2}}\left(d w^{2}+\eta_{\mu \nu} d x^{\mu} d x^{\nu}\right)+\frac{R^{3 / 2}}{U^{1 / 2}(w)} d \Omega_{4}^{2},
$$

after we trade off the holographic (or radial) coordinate $U$ in favor of a conformal one $w$ as $\# 4$

$$
w=\int_{U_{K K}}^{U} R^{3 / 2} d U^{\prime} / \sqrt{U^{\prime 3}-U_{K K}^{3}},
$$

which resides in a finite interval of length $\sim O\left(1 / M_{K K}\right)$ where $M_{K K} \equiv 3 U_{K K}^{1 / 2} / 2 R^{3 / 2}$. Thus, the topology of the $D 8$ worldvolume is $R^{3+1} \times I \times S^{4}$. The nominal Yang-Mills coupling $g_{Y M}^{2}$ is related to the other parameters as

$$
g_{Y M}^{2}=2 \pi g_{s} M_{K K} l_{s} .
$$

\#4 This $w$ coordinate is related to another convenient choice of radial coordinate $z$

$$
U^{3}=U_{K K}^{3}+U_{K K} z^{2}
$$

as

$$
d\left(w M_{K K}\right)=\left(\frac{U_{K K}}{U}\right)^{2} \frac{d z}{U_{K K}}=\frac{1}{\left(1+z^{2} / U_{K K}^{2}\right)^{2 / 3}} d\left(z / U_{K K}\right) .
$$


The low energy parameters of this holographic theory are $M_{K K}$ and $\lambda$, which together with $N_{c}$ set all the physical scales such as the QCD scale and the pion decay constant.

In the low energy limit, this is reduced to a five-dimensional Yang-Mills theory with a Chern-Simons term

$$
-\frac{1}{4} \int_{4+1} \frac{1}{e(w)^{2}} \sqrt{-h_{4+1}} \operatorname{tr} \mathcal{F}^{2}+\frac{N_{c}}{24 \pi^{2}} \int_{4+1} \omega_{5}(\mathcal{A}),
$$

where the position-dependent Yang-Mills coupling of this flavor gauge theory is

$$
\frac{1}{e(w)^{2}}=\frac{e^{-\Phi} V_{S^{4}}}{2 \pi\left(2 \pi l_{s}\right)^{5}}=\frac{\lambda N_{c}}{108 \pi^{3}} M_{K K} \frac{U(w)}{U_{K K}},
$$

with $V_{S^{4}}$ the position-dependent volume of $S^{4}$. The Chern-Simons coupling with $d \omega_{5}(\mathcal{A})=\operatorname{tr} \mathcal{F}^{3}$ arises because $\int_{S^{4}} d C_{3} \sim N_{c}$.

The usual Kaluza-Klein reduction results in an infinite number of vector fields, whose action can be derived explicitly as

$$
\int d x^{4} \mathcal{L}=\int d x^{4} \sum_{n \geq 1} \operatorname{tr}\left\{\frac{1}{2} \mathcal{F}_{\mu \nu}^{(n)} \mathcal{F}^{\mu \nu(n)}+m_{(n)}^{2} v_{\mu}^{(n)} v^{\mu(n)}\right\}+\cdots
$$

with $\mathcal{F}_{\mu \nu}^{(n)}=\partial_{\mu} v_{\nu}^{(n)}-\partial_{\nu} v_{\mu}^{(n)}$. When we decomposed $U\left(N_{F}\right)$ into $S U\left(N_{F}\right)$ and $U(1)$, the natural gauge generators are normalized as $\operatorname{tr} T^{2}=1 / 2$, which explains $1 / 2$ in front of the kinetic term \#5 These fields can be seen as non-zero modes in the decomposition of the gauge field, which in the (somewhat illegal but convenient) axial gauge $\mathcal{A}_{w}=0$ is

$$
\mathcal{A}_{\mu}(x ; w)=i \alpha_{\mu}(x) \psi_{(0)}(w)+i \beta_{\mu}(x)+\sum_{n \geq 1} v_{\mu}^{(n)}(x) \psi_{(n)}(w) .
$$

The eigenfunctions $\psi_{(n)}$ obey the orthonormality conditions,

$$
\int d w \frac{1}{2 e(w)^{2}} \psi_{(n)}(w)^{*} \psi_{(m)}(w)=\delta_{n m},
$$

for $n, m \geq 1$. For later purpose, it is useful to introduce

$$
\hat{\psi}_{(n)}(\hat{w})=\sqrt{\frac{216 \pi^{3}}{\lambda N_{c}}} \psi_{(n)}(w)
$$

\footnotetext{
${ }^{\# 5}$ In the published and all prior versions of Ref. [6], the kinetic terms of vector and axial-vector mesons were normalized with $1 / 4$ in front of the kinetic term before the trace. With canonical normalization for (axial-)vector mesons, the cubic couplings involving a vector or an axial-vector meson there should be all multiplied by $\sqrt{2}$.
} 
whose form is insensitive to $\lambda N_{c}$. Here and in what follows, $\hat{w} \equiv w M_{K K}$. Because $\mathcal{A}$ has a specific parity, the parity of $v_{n}$ 's are determined by the parity of the eigenfunctions $\psi_{(n)}(w)$ along the fifth direction. Since the parity of any one-dimensional eigenvalue system alternates, an alternating tower of vector and axial-vector fields emerge as the masses $m_{(n)}$ of the KK modes increase.

To understand this zero mode part, captured in part by the nonnormalizable eigenfunction, $\psi_{(0)}$. it is better to give up the axial gauge and consider the Wilson line,

$$
U(x)=e^{i \int_{w} \mathcal{A}(x, w)},
$$

which, as the notation suggests, one identifies with the pion field $U(x)=e^{2 i \pi(x) / f_{\pi}}$. Upon taking a singular gauge transformation back to $\mathcal{A}_{w}=0$, one finds that it is related to $\alpha$ and $\beta$ as

$$
\alpha_{\mu}(x) \equiv\left\{U^{-1 / 2}, \partial_{\mu} U^{1 / 2}\right\}, \quad 2 \beta_{\mu}(x) \equiv\left[U^{-1 / 2}, \partial_{\mu} U^{1 / 2}\right] .
$$

Truncating to this zero mode sector reproduces a Skyrme Lagrangian of pions [16] as a dimensional reduction of the five-dimensional Yang-Mills action,

$$
\int d x^{4}\left(\frac{f_{\pi}^{2}}{4} \operatorname{tr}\left(U^{-1} \partial_{\mu} U\right)^{2}+\frac{1}{32 e_{\text {Skyrme }}^{2}} \operatorname{tr}\left[U^{-1} \partial_{\mu} U, U^{-1} \partial_{\nu} U\right]^{2}\right),
$$

with $f_{\pi}^{2}=\left(g_{Y M}^{2} N_{c}\right) N_{c} M_{K K}^{2} / 54 \pi^{4}$ and $1 / e_{\text {Skyrme }}^{2} \simeq 61\left(g_{Y M}^{2} N_{c}\right) N_{c} / 54 \pi^{7}$. No other quartic terms arise, nor do we find higher order terms in derivative, although we do recover the Wess-Zumino-Witten term from the Chern-Simons term [1]. To compare against actual QCD, we must fix $\lambda=g_{Y M}^{2} N_{c} \simeq 17$ and $M_{K K} \simeq 0.94 \mathrm{GeV}$ to fit both the pion decay constant $f_{\pi}$ and the mass of the first vector meson.

\section{Holographic Baryons}

The five-dimensional effective action for the $U\left(N_{F}\right)$ gauge field in Eq. (2.7) admits solitons which carry a Pontryagin number

$$
\frac{1}{8 \pi^{2}} \int_{R^{3} \times I} \operatorname{tr} F \wedge F=k
$$

with integral $k$. We denoted by $F$ the non-Abelian part of $\mathcal{F}$ (and similarly later, $A$ for the non-Abelian part of $\mathcal{A}$ ). The smallest unit with $k=1$ carries quantum numbers of the unit baryon.

The easiest way to see this identification is to relate it to the Skyrmion [16] of chiral perturbation theory, which is the natural object in the large $N_{c}$ limit [17] of QCD. Recall that both instantons and Skyrmions are labeled by the third homotopy 
group $\pi_{3}$ of a group manifold, which is the integer for any semi-simple Lie group manifold $G$. For the Skyrmion, the winding number shows up in the classification of maps

$$
U(x): R^{3} \rightarrow S U\left(N_{F}=2\right),
$$

while for the instanton it shows up as winding number at infinity,

$$
A(x, w \rightarrow \pm \infty)=i g_{ \pm}(x)^{\dagger} d g_{ \pm}(x),
$$

with

$$
g_{-}(x)^{\dagger} g_{+}(x): R^{3} \rightarrow S U\left(N_{F}\right) .
$$

The relationship between the two types of the soliton is immediate [18] once we identify

$$
U(x)=g_{-}(x)^{\dagger} g_{+}(x) .
$$

Therefore, the instanton soliton in five dimensions is the holographic image of the Skyrmions in four dimensions. We will call it the instanton soliton.

Unlike the usual Yang-Mills theory in flat $R^{4}$ background, the effective action has a position-dependent inverse Yang-Mills coupling $1 / e(w)^{2}$ which is a monotonically increasing function of $|w|$. Since the Pontryagin density contributes to the action as multiplied by $1 / e(w)^{2}$, this tends to position the soliton near $w=0$ and also shrink it for the same reason. The $F^{2}$ energy of a trial configuration with size $\rho$ can be estimated easily in the small $\rho$ limit \#6

$$
E_{\text {Pontryagin }}=\frac{\lambda N_{c}}{27 \pi} M_{K K} \times\left(1+\frac{1}{6} M_{K K}^{2} \rho^{2}+\cdots\right),
$$

which clearly shows that the energy from the kinetic term increases with $\rho$. This by itself would collapse the soliton to a point-like one, making further analysis impossible.

A second difference comes from the presence of the additional Chern-Simons term $\sim \operatorname{tr} \mathcal{A} \wedge \mathcal{F} \wedge \mathcal{F}$, whereby the Pontryagin density $F \wedge F$ sources some of the gauge field $\mathcal{A}$ minimally. This electric charge density costs the Coulombic energy

$$
E_{\text {Coulomb }} \simeq \frac{1}{2} \times \frac{e(0)^{2} N_{c}^{2}}{10 \pi^{2} \rho^{2}}+\cdots,
$$

again in the limit of $\rho M_{K K} \ll 1$. This Coulombic energy tends to favor larger soliton size, which competes against the shrinking force due to $E_{\text {Pontryagin }}$.

The combined energy is minimized at [4, 5, 6]

$$
\rho_{\text {baryon }} \simeq \frac{\left(2 \cdot 3^{7} \cdot \pi^{2} / 5\right)^{1 / 4}}{M_{K K} \sqrt{\lambda}},
$$

\#6 The estimate of energy here takes into account the spread of the instanton density $D\left(x^{i}, w\right) \sim$ $\rho^{4} /\left(r^{2}+w^{2}+\rho^{2}\right)^{4}$, but ignores the deviation from the flat geometry along the four spatial directions. 
and the classical mass of the stabilized soliton is

$$
\begin{aligned}
m_{B}^{\text {classical }} & =\left.\left(E_{\text {Pontryagin }}+E_{\text {Coulomb }}\right)\right|_{\text {minimum }} \\
& =\frac{\lambda N_{c}}{27 \pi} M_{K K} \times\left(1+\frac{\sqrt{2 \cdot 3^{5} \cdot \pi^{2} / 5}}{\lambda}+\cdots\right) .
\end{aligned}
$$

As was mentioned above, the size $\rho_{\text {baryon }}$ is significantly smaller than $\sim 1 / M_{K K}$. We have a classical soliton whose size is a lot smaller than the fundamental scale of the effective theory \#7

For the sake of simplicity, and also because the quarks in this model have no bare mass, we will take $N_{F}=2$ for the rest of the note. A unit instanton soliton in question comes with six collective coordinates. Three correspond to the position in $R^{3}$, and three correspond to the gauge angles in $S U\left(N_{F}=2\right)$. If the soliton is small enough $\left(\rho M_{K K} \ll 1\right)$, there exists approximate symmetries $S O(4)=S U(2)_{+} \times S U(2)_{-}$at $w=0$, so the total rotational symmetry of a small solution at origin is $\operatorname{SU}\left(N_{F}=\right.$ $2) \times S U(2)_{+} \times S U(2)_{-}$. The instanton can be rotated by a conjugate $S U(2)$ action as,

$$
F \quad \rightarrow \quad S^{\dagger} F S
$$

with any $2 \times 2$ special unitary matrices $S$ which span $\mathbf{S}^{3}$. Then, the quantization of the soliton is a matter of finding eigenstates of free and nonrelativistic nonlinear sigma-model onto $\mathbf{S}^{3}$ [20, 21]. Under the approximate symmetry $S U\left(N_{F}=2\right) \times$ $S U(2)_{+} \times S U(2)_{-}$, the quantized instantons are in [22]

$$
(2 s+1 ; 2 s+1 ; 1),
$$

while the quantized anti-instantons are in

$$
(2 s+1 ; 1 ; 2 s+1) .
$$

Possible values for $s$ are integers and half-integers. However, we are eventually interested in $N_{c}=3$, in which case spins and isospins are naturally half-integral. Thus we will subsequently consider the case of $s=1 / 2$ states only, which are nucleons. Exciting these isospin comes at energy cost.

\footnotetext{
\#7 This tendency of the baryonic soliton shrinking to smaller size can be understood as being due to the backreaction of vector and axial vector mesons on the conventional Skyrmion [19.
} 


\section{Nucleon-Meson Interactions from Holography}

\subsection{General Formulation}

The starting point is the five-dimensional effective action of isospin $1 / 2$ baryons. With

$$
\gamma^{0}=\left(\begin{array}{rr}
0 & -1 \\
1 & 0
\end{array}\right), \quad \gamma^{i}=\left(\begin{array}{cc}
0 & \sigma_{i} \\
\sigma_{i} & 0
\end{array}\right), \quad \gamma^{5}=\left(\begin{array}{rr}
1 & 0 \\
0 & -1
\end{array}\right)
$$

we have the following five-dimensional effective action,

$$
\begin{aligned}
& \int d^{4} x d w\left[-i \overline{\mathcal{B}} \gamma^{m} D_{m} \mathcal{B}-i m_{\mathcal{B}}(w) \overline{\mathcal{B}} \mathcal{B}+\frac{2 \pi^{2} \rho_{\text {baryon }}^{2}}{3 e^{2}(w)} \overline{\mathcal{B}} \gamma^{m n} F_{m n} \mathcal{B}\right] \\
- & \int d^{4} x d w \frac{1}{4 e^{2}(w)} \operatorname{tr} \mathcal{F}_{m n} \mathcal{F}^{m n},
\end{aligned}
$$

where the covariant derivative is defined as $D_{m}=\partial_{m}-i\left(N_{c} \mathcal{A}_{m}^{U(1)}+A_{m}\right)$ with $A_{m}$ in the fundamental representation of $S U\left(N_{F}=2\right)$. The position-dependent mass $m_{\mathcal{B}}(w) \simeq 4 \pi^{2} / e(w)^{2} \times(1+O(1 / \lambda))$ is a very sharp increasing function of $|w|$, such that in the large $N_{c}$ and large $\lambda$ limit, the baryon wavefunction is effectively localized at $w=0$. This is the limit where the above effective action is trustworthy.

The vertex $\overline{\mathcal{B}} F \mathcal{B}$ has the coefficient function, about which we only know the central value precisely as

$$
\frac{2 \pi^{2} \rho_{\text {baryon }}^{2}}{3 e^{2}(0)}=\frac{N_{c}}{\sqrt{30}} \cdot \frac{1}{M_{K K}},
$$

which shows that this second interaction vertex can be actually dominant over the minimal coupling, although it looks subleading in the derivative expansion. As it turns out, this term is dominant for cubic vertex processes involving pions or axial vector mesons [6]. How to continue this coefficient function to $w \neq 0$ is unknown. However, for all large $\lambda N_{c}$ estimate of nucleon-meson interaction terms, only this central value matters. We chose to use the specific form above for a later convenience but it is important to remind ourselves that the precise choice does not matter.

To obtain interactions between nucleons and mesons, we mode expand $\mathcal{B}\left(x^{\mu}, w\right)=$ $B_{+}\left(x^{\mu}\right) f_{+}(w)+B_{-}\left(x^{\mu}\right) f_{-}(w)$ where $\gamma^{5} B_{ \pm}= \pm B_{ \pm}$and the profile functions $f_{ \pm}(w)$ satisfy

$$
\begin{aligned}
\partial_{w} f_{+}(w)+m_{\mathcal{B}}(w) f_{+}(w) & =m_{\mathcal{N}} f_{-}(w), \\
-\partial_{w} f_{-}(w)+m_{\mathcal{B}}(w) f_{-}(w) & =m_{\mathcal{N}} f_{+}(w),
\end{aligned}
$$

in the range $w \in\left[-w_{\max }, w_{\max }\right]$. The $4 \mathrm{D}$ Dirac field for the nucleon is then reconstructed as

$$
\mathcal{N}=B_{+}+B_{-},
$$


The eigenvalue $m_{\mathcal{N}}$ is the mass of the nucleon mode $\mathcal{N}(x)$. Approximating $m_{\mathcal{B}}(w) \simeq$ $m_{B}^{\text {classical }}\left(1+\left(w M_{K K}\right)^{2} / 3+\cdots\right)$, we find

$$
m_{\mathcal{N}} \simeq m_{B}^{\text {classical }}+O\left(M_{K K}\right)
$$

so for large $\lambda$ and large $N_{c}$ limit, we can take $m_{\mathcal{N}} \simeq m_{B}^{\text {classical }} \simeq \lambda N_{c} M_{K K} / 27 \pi$. The eigenfunctions $f_{ \pm}(w)$ are also normalized as

$$
\int_{-w_{\max }}^{w_{\max }} d w\left|f_{+}(w)\right|^{2}=\int_{-w_{\max }}^{w_{\max }} d w\left|f_{-}(w)\right|^{2}=1
$$

Note that there is a 1-1 mapping of eigenmodes with $f_{-}(w)= \pm f_{+}(-w)$, where the sign choice is tied to the sign choice for $m_{\mathcal{N}}$. Due to the asymmetry under $w \rightarrow-w$, $f_{+}(w)$ tends to shift to the positive $w$ side, and the opposite happens for $f_{-}(w)$. In this note, we will take the convention where $f_{-}(w)=f_{+}(-w)$. Both can be taken to be real.

Inserting this into the action (4.2), we find the following structure of the fourdimensional nucleon action

$$
\int d x^{4} \mathcal{L}_{4}=\int d x^{4}\left(-i \overline{\mathcal{N}} \gamma^{\mu} \partial_{\mu} \mathcal{N}-i m_{\mathcal{N}} \overline{\mathcal{N}} \mathcal{N}+\mathcal{L}_{\text {vector }}+\mathcal{L}_{\text {axial }}\right)
$$

where we have, schematically, the vector-like cubic couplings

$$
\mathcal{L}_{\text {vector }}=-i \overline{\mathcal{N}} \gamma^{\mu} \beta_{\mu} \mathcal{N}-\sum_{k \geq 1} g_{V}^{(k)} \overline{\mathcal{N}} \gamma^{\mu} v_{\mu}^{(2 k-1)} \mathcal{N}+\sum_{k \geq 1} g_{d V}^{(k)} \overline{\mathcal{N}} \gamma^{\mu \nu} \partial_{\mu} v_{\nu}^{(2 k-1)} \mathcal{N}
$$

and the axial cubic couplings to axial mesons,

$$
\mathcal{L}_{\text {axial }}=-\frac{i g_{A}}{2} \overline{\mathcal{N}} \gamma^{\mu} \gamma^{5} \alpha_{\mu} \mathcal{N}-\sum_{k \geq 1} g_{A}^{(k)} \overline{\mathcal{N}} \gamma^{\mu} \gamma^{5} v_{\mu}^{(2 k)} \mathcal{N}+\sum_{k \geq 1} g_{d A}^{(k)} \overline{\mathcal{N}} \gamma^{\mu \nu} \gamma^{5} \partial_{\mu} v_{\nu}^{(2 k)} \mathcal{N}
$$

For instance, $g_{A}$ is the axial coupling to pions, whose leading cubic coupling to $\mathcal{N}$ appears via

$$
\alpha_{\mu}^{S U(2)}=\left\{\xi^{-1}, \partial_{\mu} \xi\right\}^{S U(2)}=\frac{2 i}{f_{\pi}} \partial_{\mu} \pi+\cdots=\frac{2 i}{f_{\pi}} \partial_{\mu} \pi^{a} \frac{\tau^{a}}{2}+\cdots
$$

We will ignore quartic couplings involving more than one spin 1 mesons.

We must recall an important detail which is suppressed in the notation above, regarding the differences between the isospin singlet mesons and the triplet mesons. These two are packaged into the five-dimensional gauge field $\mathcal{A}$ as the trace part and the $S U(2)$ part, respectively. For instance, the vector mesons would show up in $\mathcal{A}$ as

$$
v_{\mu}^{(2 k-1)}=\left(\begin{array}{cc}
1 / 2 & 0 \\
0 & 1 / 2
\end{array}\right) \omega_{\mu}^{(k)}+\rho_{\mu}^{(k) a} \frac{\tau^{a}}{2}
$$


where $\omega$ 's and $\rho$ 's are canonically normalized. A crucial point is that the representation of $\mathcal{A}$ that appears in the baryon effective action is different from this. Instead, the vector meson that enters the baryon vertex has the form

$$
v_{\mu}^{(2 k-1)}=\left(\begin{array}{cc}
N_{c} / 2 & 0 \\
0 & N_{c} / 2
\end{array}\right) \omega_{\mu}^{(k)}+\rho_{\mu}^{(k) a} \frac{\tau^{a}}{2},
$$

implying the isosinglet has a relative enhancement factor of $N_{c}$. The second difference can be seen in the fact that only the isotriplets appear in the $\overline{\mathcal{B}} F \mathcal{B}$ vertex in five dimensions. In any case, each and every cubic coupling above comes in two different varieties, ones for isosinglet mesons, such as $\eta$ and $\omega$, and those for isotriplet mesons, such as $\pi$ and $\rho$.

\subsection{Structure of the Cubic Couplings}

All the coupling constants $g_{V, A}^{(k)}, g_{d V, d A}^{(k)}$, and $g_{A}$ are calculated by suitable wave-function overlap integrals involving $f_{ \pm}$and $\psi_{(n)}$ 's [6] $\# 8$ Let us consider the general structure. Contributions from the minimal coupling, $\overline{\mathcal{B}} \gamma^{\mu} \mathcal{A}_{\mu} \mathcal{B}$, has the form,

$$
A_{n}^{ \pm} \equiv \int_{-w_{\max }}^{w_{\max }} d w\left|f_{ \pm}(w)\right|^{2} \psi_{(n)}(w)
$$

$A_{n}^{ \pm}$'s contribute to dimension four vertices, most notably $\overline{\mathcal{N}} \gamma^{\mu} \rho_{\mu} \mathcal{N}, \overline{\mathcal{N}} \gamma^{\mu} \omega_{\mu} \mathcal{N}$, and their axial vector counterparts. They also contribute to $\overline{\mathcal{N}} \gamma^{\mu} \gamma^{5} \partial_{\mu} \pi \mathcal{N}$, although only as a subleading contribution.

Contributions from $\overline{\mathcal{B}} F \mathcal{B}$ have the general forms

$$
B_{n}^{ \pm} \equiv \int_{-w_{\max }}^{w_{\max }} d w\left(\frac{2 \pi^{2} \rho_{\text {baryon }}^{2}}{3 e(w)^{2}}\right) f_{\mp}(w)^{*} f_{ \pm}(w) \psi_{(n)}(w),
$$

for $\overline{\mathcal{B}} \gamma^{\mu \nu} F_{\mu \nu} \mathcal{B}$, and

$$
C_{n}^{ \pm} \equiv \int_{-w_{\max }}^{w_{\max }} d w\left(\frac{2 \pi^{2} \rho_{\text {baryon }}^{2}}{3 e(w)^{2}}\right)\left|f_{ \pm}(w)\right|^{2} \partial_{w} \psi_{(n)}(w),
$$

for $\overline{\mathcal{B}} \gamma^{5 \mu} F_{5 \mu} \mathcal{B}$. The latter two sets contribute only to the isotriplets. $B_{n}^{ \pm}$'s contribute to the derivative couplings such as $\overline{\mathcal{N}} \gamma^{\mu \nu} \partial_{\mu} \rho_{\nu} \mathcal{N}$. $C_{n}^{ \pm}$'s generate the large $N_{c}$ leading contributions to vertices involving isotriplet axial mesons, such as $\overline{\mathcal{N}} \gamma^{\mu} \gamma^{5} \partial_{\mu} \pi \mathcal{N}$ and the minimal coupling to the axial vectors $\overline{\mathcal{N}} \gamma^{\mu} \gamma^{5} a_{\mu} \mathcal{N}$. C's also contribute subleading pieces to vertices like $\overline{\mathcal{N}} \gamma^{\mu} \rho_{\mu} \mathcal{N}$.

\footnotetext{
${ }^{\# 8} \mathrm{~A}$ related but different approach to these couplings was later formulated in Ref. 23] which adopted the conventional methods used for Skyrmions. Since both are based on the classical solitons quantized over the moduli space, the end results should be equivalent.
} 
From these, we have the following cubic couplings for isospin triplet mesons,

$$
\begin{aligned}
g_{A}^{\text {triplet }} & =4 C_{0}^{+}+2 A_{0}^{+}, \\
g_{V}^{(k) \text { triplet }} & =A_{2 k-1}^{+}+2 C_{2 k-1}^{+}, \\
g_{A}^{(k) \text { triplet }} & =2 C_{2 k}^{+}+A_{2 k}^{+}, \\
g_{d V}^{(k) \text { triplet }} & =2 B_{2 k-1}^{+}, \\
g_{d A}^{(k) \text { triplet }} & =2 B_{2 k}^{+},
\end{aligned}
$$

where the mesons in these vertices are in the form such as $\alpha_{\mu}^{(S U(2)}=2 i / f_{\pi} \times\left(\partial_{\mu} \pi^{a} \tau^{a} / 2\right)$ and $\rho_{\mu}^{a} \tau^{a} / 2$. As we will see shortly, the second terms for the first three lines are subleading in the $\lambda N_{c}$ limit, so that we actually have $g_{A}^{\text {triplet }} \simeq 4 C_{0}^{+}, g_{V}^{(k) \text { triplet }} \simeq A_{2 k-1}^{+}$, $g_{A}^{(k) \text { triplet }} \simeq 2 C_{2 k}^{+}$. Of these, $g_{d A}^{(k) \text { triplet }}=0$ identically, implying that axial vectors have no derivative coupling in our approximation.

For isospin singlets, $B$ and $C$ contributions are absent so we have

$$
\begin{aligned}
g_{A}^{\text {singlet }} & =2 A_{0}^{+}, \\
g_{V}^{(k) \text { singlet }} & =A_{2 k-1}^{+}, \\
g_{A}^{(k) \text { singlet }} & =A_{2 k}^{+} .
\end{aligned}
$$

The mesons in these vertices are in the form such as $\alpha_{\mu}^{U(1)}=2 i / f_{\pi} \times\left(\left(N_{c} / 2\right) \partial_{\mu} \eta^{\prime}\right)$ and $\left(N_{c} / 2\right) \omega_{\mu}$.

Remarkably, even before we go into any detail, we have a prediction that all isospin singlet vectors and all axial-vectors have no derivative coupling in this approximation.

\subsection{Scaling of the Cubic Couplings}

The key fact that allows us to extract large $\lambda N_{c}$ behavior of cubic couplings is that $m_{\mathcal{B}}(w) \sim 1 / e(w)^{2}$ is proportional to $\lambda N_{c}$. Relative to the mesonic eigenfunctions $\psi_{(n)}, f_{ \pm}$becomes more and more concentrated at $w=0$. The two wavefunctions are slightly off-set from the center by the amount $\sim \pm 1 /\left(M_{K K} \lambda N_{c}\right)$ with the width of order $\sim 1 /\left(M_{K K} \sqrt{\lambda N_{c}}\right)$. This allows us to approximate $f_{ \pm}^{2}$ or $f_{+} f_{-}$by a delta function at origin in wavefunction overlap integrals such as $A_{n}^{ \pm}$provided that the integrand does not vanish near $w=0$.

For example, it is easy to see that

$$
A_{2 k-1}^{ \pm} \rightarrow \psi_{(2 k-1)}(0)=\sqrt{\frac{216 \pi^{3}}{\lambda N_{c}}} \hat{\psi}_{(2 k-1)}(\hat{w}=0)
$$


in the large $\lambda N_{c}$ limit. Here we also used the fact that $\hat{\psi}_{(n)}\left(\hat{w}=w M_{K K}\right)$ for $n \geq 1$ obey

$$
\int d \hat{w} \frac{e^{2}(0)}{e^{2}(w)} \hat{\psi}_{(n)}(\hat{w}) \hat{\psi}_{(m)}(\hat{w})=\delta_{n m}
$$

and are independent of $\lambda N_{c}$ and of $M_{K K}$. In particular, numerically we find $\hat{\psi}_{(1)}(0) \simeq$ 0.597. This number is an important ingredient of the low energy nucleon-nucleon potential as we will find later.

$A_{2 k}^{ \pm}$'s, whose integrands vanish at $w=0$, take more care. Using reality and the eigenmode equation for $f_{ \pm}$,

$$
A_{2 k}^{ \pm}=\frac{1}{m_{\mathcal{N}}} \int_{w} f_{ \pm}(w)\left(\mp \partial_{w} f_{\mp}(w)+m_{\mathcal{B}}(w) f_{\mp}(w)\right) \psi_{(2 k)}(w) .
$$

Since $\psi_{(2 k)}$ is odd, the leading contribution arises from the derivative piece, and we find

$$
A_{2 k}^{ \pm} \rightarrow \pm \frac{M_{K K}}{2 m_{\mathcal{N}}} \sqrt{\frac{216 \pi^{3}}{\lambda N_{c}}} \hat{\psi}_{(2 k)}{ }^{\prime}(\hat{w}=0),
$$

which scales as $1 /\left(\lambda N_{c}\right)^{3 / 2}$. Note that $\left|A_{2 k-1}\right| \sim\left(\lambda N_{c}\right)^{-1 / 2} \gg\left|A_{2 k}\right| \sim\left(\lambda N_{c}\right)^{-3 / 2}$.

Evaluation of $B$ 's is simpler because it involves $f_{+} f_{-}$which is an even function, so that

$$
B_{n}^{ \pm}=\frac{2 \pi^{2} \rho_{\text {baryon }}^{2}}{3} \int_{w} \frac{1}{e(w)^{2}} f_{\mp}(w)^{*} f_{ \pm}(w) \psi_{(n)}(w) .
$$

With even $\psi$ 's, this gives

$$
B_{2 k-1}^{ \pm} \rightarrow \frac{2 \pi^{2} \rho_{\text {baryon }}^{2}}{3 e(0)^{2}} \sqrt{\frac{216 \pi^{3}}{\lambda N_{c}}} \hat{\psi}_{(2 k-1)}(0)
$$

whereas the odd cases vanish identically

$$
B_{2 k}^{ \pm}=0 \text {. }
$$

Evaluation of $C_{n}^{ \pm}$proceeds similarly as $A_{n}^{ \pm}$. Using the equation of motion for $f_{ \pm}$ again and recalling that

$$
\frac{2 \pi^{2} \rho_{\text {baryon }}^{2}}{3 e(w)^{2}} \simeq \frac{\rho_{\text {baryon }}^{2}}{6} \times m_{\mathcal{B}}(w),
$$

we find

$$
C_{n}^{ \pm}=\frac{\rho_{\text {baryon }}^{2}}{6} \int_{w} f_{ \pm}\left(\mp \partial_{w} f_{ \pm}+m_{\mathcal{N}} f_{\mp}\right) \partial_{w} \psi_{(n)}(w) .
$$


This gives

$$
\begin{aligned}
C_{2 k-1}^{ \pm} & \rightarrow \pm \frac{\rho_{\text {baryon }}^{2}}{12} M_{K K}^{2} \sqrt{\frac{216 \pi^{3}}{\lambda N_{c}}} \hat{\psi}_{(2 k-1)}{ }^{\prime \prime}(0) \\
C_{2 k}^{ \pm} & \rightarrow \frac{\rho_{\text {baryon }}^{2}}{6} m_{\mathcal{N}} M_{K K} \sqrt{\frac{216 \pi^{3}}{\lambda N_{c}}} \hat{\psi}_{(2 k)}{ }^{\prime}(0)
\end{aligned}
$$

Also note that $\left|C_{2 k}\right| \sim \sqrt{N_{c} / \lambda} \gg\left|C_{2 k-1}\right| \sim 1 / \sqrt{\lambda^{3} N_{c}}$.

The case of $n=0$ requires special attention since $\psi_{(0)}(w)$ is not normalizable and only its derivative, which is normalizable, appears in the physical quantities. The conventional choice is such that $\partial_{\hat{w}} \psi_{(0)}(0)=1 / \pi$, which is necessary for the familiar chiral Lagrangian to emerge from this formulation. With this, we find

$$
A_{0}^{ \pm} \rightarrow \pm \frac{M_{K K}}{2 m_{\mathcal{N}}} \frac{1}{\pi}
$$

and

$$
B_{0}^{ \pm}=0
$$

Finally, with the specific functional form $\sim 1 / e(w)^{2}$ of the $\overline{\mathcal{B}} F \mathcal{B}$ coefficient, we have an analytical result,

$$
C_{0}^{ \pm}=\frac{\rho_{\text {baryon }}^{2} m_{\mathcal{N}} M_{K K}}{6 \pi}=\frac{N_{c}}{\sqrt{30}} \frac{1}{\pi} .
$$

These enter pion-nucleon couplings, which come with additional factors of $1 / f_{\pi}$ for each pion.

Note that some of the above integrals have signs sensitive to the choice of $f_{ \pm}$. Since $f_{ \pm}$are wavefunctions specific to the chiral and the anti-chiral spinors, these \pm signs for the values of $A_{2 k}^{ \pm}$and $C_{2 k-1}^{ \pm}$have the net effective of introducing a $\gamma^{5}$ to the vertex as a part of dimensional reduction process, in addition to the existing Dirac matrices of the vertices in (4.2). This is already manifest in how these coefficients contributes to the cubic couplings in Eq. (4.17),(4.18).

\subsection{Pseudo-scalar Mesons: $\pi$ and $\eta^{\prime}$}

Starting with

$$
-\frac{i g_{A}}{2} \overline{\mathcal{N}} \gamma^{\mu} \gamma^{5} \alpha_{\mu} \mathcal{N}
$$

we restore the isotriplet and the isosinglet mesons and find

$$
\frac{g_{A}^{\text {triplet }}}{2 f_{\pi}} \overline{\mathcal{N}} \gamma^{\mu} \gamma^{5} \partial_{\mu}\left(\pi^{a} \tau^{a}\right) \mathcal{N}+\frac{g_{A}^{\text {singlet }} N_{c}}{2 f_{\pi}} \overline{\mathcal{N}} \gamma^{\mu} \gamma^{5} \partial_{\mu} \eta^{\prime} \mathcal{N}
$$


Since we will be considering $N_{f}=2$, the distinction between $\eta$ and $\eta^{\prime}$ becomes a bit ambiguous. Here $\eta^{\prime}$ denotes the trace part of the pseudo-scalar, regardless of the number of flavors. In turn, this is equivalent to

$$
-\left(\frac{g_{A}^{\text {triplet }}}{2 f_{\pi}} \times 2 m_{\mathcal{N}}\right) \overline{\mathcal{N}} \gamma^{5}\left(\pi^{a} \tau^{a}\right) \mathcal{N}-\left(\frac{g_{A}^{\text {singlet }} N_{c}}{2 f_{\pi}} \times 2 m_{\mathcal{N}}\right) \overline{\mathcal{N}} \gamma^{5} \eta^{\prime} \mathcal{N}
$$

\subsection{Vector Mesons: $\rho$ and $\omega$}

We will denote the isotriplet vectors by $\rho^{(k)}$ and singlets by $\omega^{(k)}$, upon which

$$
-\sum_{k \geq 1} g_{V}^{(k)} \overline{\mathcal{N}} \gamma^{\mu} v_{\mu}^{(2 k-1)} \mathcal{N}+\sum_{k \geq 1} g_{d V}^{(k)} \overline{\mathcal{N}} \gamma^{\mu \nu} \partial_{\mu} v_{\nu}^{(2 k-1)} \mathcal{N}
$$

separates to

$$
-\sum_{k \geq 1}\left(\frac{g_{V}^{(k) \text { triplet }}}{2}\right) \overline{\mathcal{N}} \gamma^{\mu} \rho_{\mu}^{(k) a} \tau^{a} \mathcal{N}+\sum_{k \geq 1}\left(\frac{g_{d V}^{(k) \text { triplet }}}{2}\right) \overline{\mathcal{N}} \gamma^{\mu \nu} \partial_{\mu} \rho_{\nu}^{(k) a} \tau^{a} \mathcal{N}
$$

and

$$
-\sum_{k \geq 1}\left(\frac{N_{c} g_{V}^{(k) \text { singlet }}}{2}\right) \overline{\mathcal{N}} \gamma^{\mu} \omega_{\mu}^{(k)} \mathcal{N}
$$

since the singlet does not have the derivative coupling in this approximation.

\subsection{Axial Vector Mesons: $a$ and $f$}

Similarly, the axial vector mesons couplings

$$
-\sum_{k \geq 1} g_{A}^{(k)} \overline{\mathcal{N}} \gamma^{\mu} \gamma^{5} v_{\mu}^{(2 k)} \mathcal{N}+\sum_{k \geq 1} g_{d A}^{(k)} \overline{\mathcal{N}} \gamma^{\mu \nu} \gamma^{5} \partial_{\mu} v_{\nu}^{(2 k)} \mathcal{N}
$$

can be written as

$$
-\sum_{k \geq 1}\left(\frac{g_{A}^{(k) \text { triplet }}}{2}\right) \overline{\mathcal{N}} \gamma^{\mu} \gamma^{5} a_{\mu}^{(k) a} \tau^{a} \mathcal{N}-\sum_{k \geq 1}\left(\frac{N_{c} g_{A}^{(k) \text { singlet }}}{2}\right) \overline{\mathcal{N}} \gamma^{\mu} \gamma^{5} f_{\mu}^{(k)} \mathcal{N}
$$

since no derivative coupling exists for axial vectors in this approximation. 


\section{$5 \quad$ Large $N_{c}$ Nucleon-Nucleon Potential}

Phenomenologically the nucleon-nucleon (NN) potential is well described by one boson exchange models. The long-range part of the NN potential is mostly due to the pion exchange, while the short-range repulsion is governed by the vector meson exchange. The scalar meson exchange is responsible for the intermediate-range of the potential. The interaction Lagrangians for boson-nucleon couplings are, for pseudoscalar mesons:

$$
\mathcal{L}_{P}=-g_{\varphi \mathcal{N} \mathcal{N}} \overline{\mathcal{N}}(x) \gamma_{5} \varphi(x) \mathcal{N}(x)
$$

and for vector mesons:

$$
\mathcal{L}_{V}=-g_{v \mathcal{N} \mathcal{N}} \overline{\mathcal{N}}(x) \gamma^{\mu} v_{\mu}(x) \mathcal{N}(x)+\frac{\tilde{g}_{v \mathcal{N N}}}{2 m_{\mathcal{N}}} \overline{\mathcal{N}}(x) \gamma^{\mu \nu} \partial_{\mu} v_{\nu}(x) \mathcal{N}(x),
$$

where $m_{\mathcal{N}}$ is the nucleon mass. For the D4-D8 holographic model, we saw that the derivative coupling is absent for the isospin singlet vectors such as $\omega \# 9$ The same is true of axial vectors, so we have only [1]

$$
\mathcal{L}_{A}=-g_{a \mathcal{N N} \mathcal{N}} \overline{\mathcal{N}}(x) \gamma^{\mu} \gamma_{5} a_{\mu}(x) \mathcal{N}(x)
$$

Note that we now use the convention for isovector bosons as $\varphi=\vec{\tau} \cdot \vec{\varphi}, v=\vec{\tau} \cdot \vec{v}$, and $a=\vec{\tau} \cdot \vec{a}$.

It is useful to compare our convention to that of Ericson and Weise [12], which is our primary reference on one boson exchange potential. The Dirac matrices we

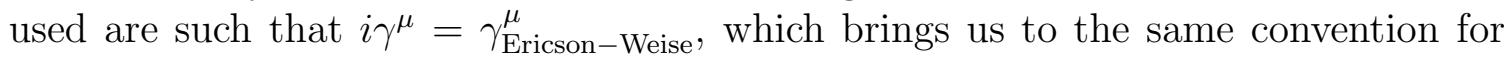
the nucleon field and its free Lagrangian. In addition, we have reversed the overall sign of the couplings from theirs as $g_{\varphi \mathcal{N N}}=-g_{P}, g_{\rho \mathcal{N N}}=-g_{V}$, and $\tilde{g}_{\rho \mathcal{N N}}=-g_{T}$, which is a matter of a common sign convention on meson fields. We have no scalar field, so do not have the counterpart of their $g_{S}$.

The leading large $N_{c}$ and large $\lambda$ scaling is such that, for pseudo-scalars $\left(\varphi=\pi, \eta^{\prime}\right)$

$$
\begin{aligned}
\frac{g_{\pi \mathcal{N N}}}{2 m_{\mathcal{N}}} M_{K K} & =\frac{g_{A}^{\text {triplet }}}{2 f_{\pi}} M_{K K} \simeq \frac{2 \cdot 3 \cdot \pi}{\sqrt{5}} \times \sqrt{\frac{N_{c}}{\lambda}} \\
\frac{g_{\eta^{\prime} \mathcal{N} \mathcal{N}}}{2 m_{\mathcal{N}}} M_{K K} & =\frac{N_{c} g_{A}^{\text {singlet }}}{2 f_{\pi}} M_{K K} \simeq \sqrt{\frac{3^{9}}{2}} \pi^{2} \times \frac{1}{\lambda N_{c}} \sqrt{\frac{N_{c}}{\lambda}}
\end{aligned}
$$

\#9 Note that, empirically, $\tilde{g} / g=3.7-6.1$ for the $\rho$-meson (see for example 9 ), while for $\omega$-mesons the ratio is close to zero, for instance $\tilde{g} / g=0.1 \pm 0.2[12$. 
for vectors $\left(v=\rho^{(k)}, \omega^{(k)}\right)$

$$
\begin{aligned}
g_{\rho^{(k) \mathcal{N N}}} & =\frac{g_{V}^{(k) \text { triplet }}}{2} \simeq \sqrt{2 \cdot 3^{3} \cdot \pi^{3}} \hat{\psi}_{(2 k-1)}(0) \times \frac{1}{N_{c}} \sqrt{\frac{N_{c}}{\lambda}} \\
g_{\omega^{(k)} \mathcal{N N}} & =\frac{N_{c} g_{V}^{(k) \text { singlet }}}{2} \simeq \sqrt{2 \cdot 3^{3} \cdot \pi^{3}} \hat{\psi}_{(2 k-1)}(0) \times \sqrt{\frac{N_{c}}{\lambda}} \\
\frac{\tilde{g}_{\rho^{(k)} \mathcal{N N}}}{2 m_{\mathcal{N}}} M_{K K} & =\frac{g_{d V}^{(k) \text { triplet }} M_{K K}}{2} \simeq \sqrt{\frac{2^{2} \cdot 3^{2} \cdot \pi^{3}}{5}} \hat{\psi}_{(2 k-1)}(0) \times \sqrt{\frac{N_{c}}{\lambda}},
\end{aligned}
$$

and for axial vectors $\left(a=a^{(k)}, f^{(k)}\right)$,

$$
\begin{aligned}
& g_{a^{(k) \mathcal{N N}}} \equiv \frac{g_{A}^{(k) \text { triplet }}}{2} \simeq \sqrt{\frac{2^{2} \cdot 3^{2} \cdot \pi^{3}}{5}} \hat{\psi}_{(2 k)}{ }^{\prime}(0) \times \sqrt{\frac{N_{c}}{\lambda}} \\
& g_{f^{(k) \mathcal{N N}}} \equiv \frac{N_{c} g_{A}^{(k) \text { singlet }}}{2} \simeq \sqrt{\frac{3^{9} \cdot \pi^{5}}{2}} \hat{\psi}_{(2 k)}{ }^{\prime}(0) \times \frac{1}{\lambda N_{c}} \sqrt{\frac{N_{c}}{\lambda}}
\end{aligned}
$$

Note that $g_{\rho \mathcal{N N}}$ and $\tilde{g}_{\rho \mathcal{N N}}$ we have derived from the D4-D8 model are of the same sign, which is consistent with experimental results.

\subsection{Holographic Nucleon-Nucleon Potential}

In general, the one-boson exchange nucleon-nucleon potential can be written as

$$
V_{\pi}+V_{\eta^{\prime}}+\sum_{k=1}^{\infty} V_{\rho^{(k)}}+\sum_{k=1}^{\infty} V_{\omega^{(k)}}+\sum_{k=1}^{\infty} V_{a^{(k)}}+\sum_{k=1}^{\infty} V_{f^{(k)}} .
$$

We now borrow results on one-boson exchange potentials from Ref. [10, 12] for various mesons, and truncate to the leading contributions in $1 / N_{c}$ and in $1 / \lambda$. (For more complete forms of one boson exchange potential, we refer to Appendix 10 of Ref. [12.) In doing so, we find that not all terms in the above expansion contribute at the leading order. The leading contributions arise from the following four classes of couplings

$$
\frac{g_{\pi \mathcal{N} \mathcal{N}} M_{K K}}{2 m_{\mathcal{N}}} \sim g_{\omega^{(k)} \mathcal{N N}} \sim \frac{\tilde{g}_{\rho^{(k)} \mathcal{N N}} M_{K K}}{2 m_{\mathcal{N}}} \sim g_{a^{(k)} \mathcal{N N}} \sim \sqrt{\frac{N_{c}}{\lambda}}
$$

whereas $g_{\eta^{\prime} \mathcal{N N}}$ is further suppressed by $1 / \lambda N_{c}$ and $g_{\rho^{(k)} \mathcal{N N}}$ by $1 / N_{c}$.

For instance, the one pion exchange potential (OPEP) would be

$$
V_{\pi}=\left(\frac{g_{\pi \mathcal{N N}}}{2 m_{\mathcal{N}}}\right)^{2} \frac{m_{\pi}^{3}}{12 \pi}\left[y_{0}\left(m_{\pi} r\right) \vec{\sigma}_{1} \cdot \vec{\sigma}_{2}+y_{2}\left(m_{\pi} r\right) S_{12}\right] \vec{\tau}_{1} \cdot \vec{\tau}_{2},
$$


where $S_{12}=3(\vec{\sigma} \cdot \hat{r})\left(\sigma_{2} \cdot \hat{r}\right)-\vec{\sigma}_{1} \cdot \vec{\sigma}_{2}$, and

$$
y_{0}(x)=\frac{e^{-x}}{x}, y_{2}(x)=\left(1+\frac{3}{x}+\frac{3}{x^{2}}\right) \frac{e^{-x}}{x} .
$$

However, since we are working in the D4-D8 model where $m_{\pi}=0$, the OPEP simplifies to

$$
V_{\pi}^{\text {holographic }}=\frac{1}{4 \pi}\left(\frac{g_{\pi \mathcal{N} \mathcal{N}} M_{K K}}{2 m_{\mathcal{N}}}\right)^{2} \frac{1}{M_{K K}^{2} r^{3}} S_{12} \vec{\tau}_{1} \cdot \vec{\tau}_{2}
$$

For the isospin singlet vector meson, namely $\omega^{(k)}$-mesons, the derivative coupling is absent and the leading large $\lambda N_{c}$ contribution is very simple,

$$
V_{\omega(k)}^{\text {holographic }}=\frac{1}{4 \pi}\left(g_{\omega^{(k)} \mathcal{N N}}\right)^{2} m_{\omega^{(k)}} y_{0}\left(m_{\omega^{(k)}} r\right) .
$$

For $\rho^{(k)}$ which are the isospin triplet vector mesons, the derivative coupling is dominant over the minimal coupling. This also simplifies the potential quite a bit as

$$
\begin{aligned}
& V_{\rho^{(k)}}^{\text {holographic }} \simeq \\
& \frac{1}{4 \pi}\left(\frac{\tilde{g}_{\rho^{(k)} \mathcal{N} \mathcal{N}} M_{K K}}{2 m_{\mathcal{N}}}\right)^{2} \frac{m_{\rho^{(k)}}^{3}}{3 M_{K K}^{2}}\left[2 y_{0}\left(m_{\rho^{(k)}} r\right) \vec{\sigma}_{1} \cdot \vec{\sigma}_{2}-y_{2}\left(m_{\rho^{(k)}} r\right) S_{12}(\hat{r})\right] \vec{\tau}_{1} \cdot \vec{\tau}(5
\end{aligned}
$$

The contribution to $V_{\rho^{(k)}}$ due to the minimal coupling $g_{\rho \mathcal{N N}}$ are suppressed by additional $1 / N_{c}$.

The potential from exchange of isospin singlet axial vectors $f^{(k)}$ is suppressed by additional $1 /\left(\lambda N_{c}\right)^{2}$ while triplet axial-vector mesons $a^{(k)}$ contributes [11]

$$
\begin{aligned}
& V_{a^{(k)}}^{\text {holographic }} \simeq \\
& \frac{1}{4 \pi}\left(g_{a^{(k)} \mathcal{N N}}\right)^{2} \frac{m_{a^{(k)}}}{3}\left[-2 y_{0}\left(m_{a^{(k)}} r\right) \vec{\sigma}_{1} \cdot \vec{\sigma}_{2}+y_{2}\left(m_{a^{(k)}} r\right) S_{12}(\hat{r})\right] \vec{\tau}_{1} \cdot \vec{\tau}_{2} .
\end{aligned}
$$

Finally note that the meson masses are all of order $M_{K K}$ and $m_{\rho^{(k)}}=m_{\omega^{(k)}}<m_{a^{(k)}}$. The vector masses and the axial vector masses alternate as $k$ increases.

\subsection{Behavior at $r \sim 1 / M_{K K} \gg 1 / \sqrt{\lambda} M_{K K}$}

When the distance in question is longer than $1 / M_{K K}$, it suffices to consider contributions from light mesons only,

$$
V_{(p)} \equiv V_{\pi}^{\text {holographic }}+\sum_{k=1}^{p}\left(V_{\rho^{(k)}}^{\text {holographic }}+V_{\omega^{(k)}}^{\text {holographic }}+V_{a^{(k)}}^{\text {holographic }}\right)
$$


where the level $p$ is determined by the short distance scale, down to which we are interested. For instance, if we are interested in distance down to $1 /\left(3 M_{K K}\right), p=10$ would suffice.

More generally, with large but finite $\lambda$, the smallest distance where one can still trust this one-boson exchange potential is when the distance is comparable to the solitonic size of the nucleon at $\sim 1 / \sqrt{\lambda} M_{K K}$. Around this scale, the current set-up, where one implicitly assumes each of the unit baryon to be intact, breaks down and one must begin to consider backreactions systematically. Thus, although the sum can formally extend to $p=\infty$, it is in practice more sensible to cut it off at $p \sim \sqrt{\lambda / 10}$, after taking into accounts various order one factors.

The relevant (large $\lambda N_{c}$ ) pion coupling is

$$
\frac{g_{\pi \mathcal{N N}}}{2 m_{\mathcal{N}}} M_{K K} \simeq 8.43 \sqrt{\frac{N_{c}}{\lambda}},
$$

while for (axial-)vector mesons we parameterize the relevant coupling as

$$
g_{\omega(k) \mathcal{N N}} \simeq \xi_{k} \sqrt{\frac{N_{c}}{\lambda}}, \quad \frac{\tilde{g}_{\rho^{(k)} \mathcal{N N}}}{2 m_{\mathcal{N}}} M_{K K} \simeq \zeta_{k} \sqrt{\frac{N_{c}}{\lambda}}, \quad g_{a^{(k)} \mathcal{N N}} \simeq \chi_{k} \sqrt{\frac{N_{c}}{\lambda}} .
$$

Coefficients, $\xi_{k}, \zeta_{k}, \chi_{k}$, are determined by $\psi_{(2 k-1)}(0)$ and $\psi_{(2 k)}^{\prime}(0)$, we list these values in the following table 1 , together with the masses (in unit of $M_{K K}$ ) of the vector and the axial vector mesons.

\begin{tabular}{c||c|c|c|c||c|c|c}
$k$ & $m_{\omega^{(k)}}=m_{\rho^{(k)}}$ & $\hat{\psi}_{(2 k-1)}(0)$ & $\xi_{k}$ & $\zeta_{k}$ & $m_{a^{(k)}}$ & $\hat{\psi}_{(2 k)}^{\prime}(0)$ & $\chi_{k}$ \\
\hline 1 & 0.818 & 0.5973 & 24.44 & 8.925 & 1.25 & 0.629 & 9.40 \\
\hline 2 & 1.69 & 0.5450 & 22.30 & 8.143 & 2.13 & 1.10 & 16.4 \\
\hline 3 & 2.57 & 0.5328 & 21.81 & 7.961 & 3.00 & 1.56 & 23.3 \\
\hline 4 & 3.44 & 0.5288 & 21.64 & 7.901 & 3.87 & 2.02 & 30.1 \\
\hline 5 & 4.30 & 0.5270 & 21.57 & 7.874 & 4.73 & 2.47 & 36.9 \\
\hline 6 & 5.17 & 0.5261 & 21.52 & 7.860 & 5.59 & 2.93 & 43.8 \\
\hline 7 & 6.03 & 0.5255 & 21.50 & 7.852 & 6.46 & 3.38 & 50.5 \\
\hline 8 & 6.89 & 0.5251 & 21.48 & 7.846 & 7.32 & 3.83 & 57.3 \\
\hline 9 & 7.75 & 0.5249 & 21.48 & 7.843 & 8.19 & 4.29 & 64.1 \\
\hline 10 & 8.62 & 0.5247 & 21.47 & 7.840 & 9.05 & 4.74 & 70.9
\end{tabular}

Table 1: Numerical results for masses and coupling constants for spin one mesons interacting with nucleons.

In figure 1 , we display the shape of the large $N_{c}$ potential with $p=10$ for the isosinglet sector with total angular momentum one and total spin one. By superselection rules, the spatial angular momentum is a mixture of 0 and 2, and effectively we have

$$
S_{12}=2, \quad \vec{\tau}_{1} \cdot \vec{\tau}_{2}=-3, \quad \vec{\sigma}_{1} \cdot \vec{\sigma}_{2}=1
$$




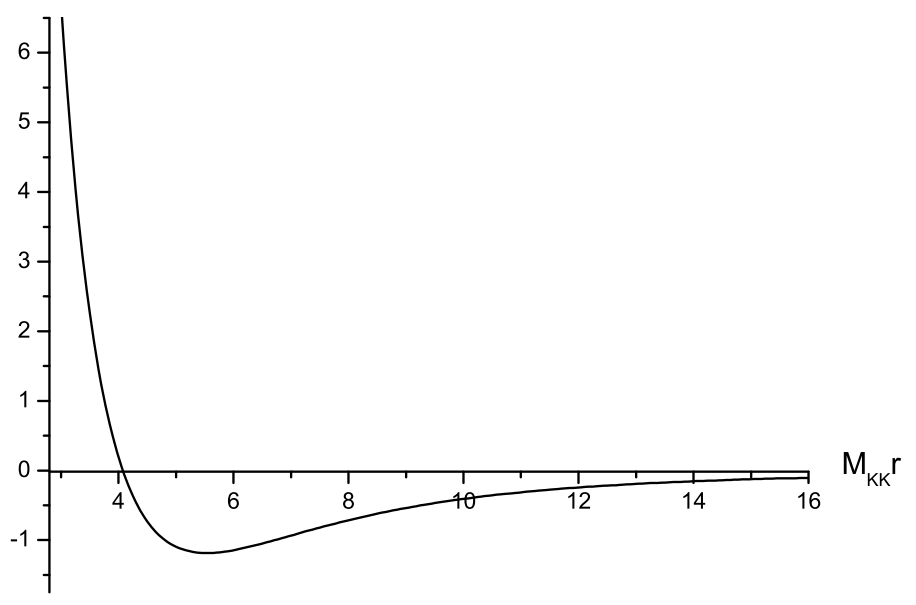

Figure 1: A plot of large $N_{c}$ nucleon-nucleon potential, truncated at $p=10$, along its most attractive channel, namely isospin singlet, $\sigma$-spin triplet, and even spatial angular momentum with $S_{12}=2$. The horizontal axis is for the distance, $r M_{K K}$, while the potential energy along the vertical axis is in unit of $M_{K K} N_{c} / 4 \pi \lambda$.

This is the only channel which is attractive at long distance. All other channels are repulsive. See section 7 for more discussion. The minimum of the potential is located around $5.5 / M_{K K}$ which is a little larger than one fermi if we adopt $M_{K K} \simeq 0.94 \mathrm{GeV}$. Toward $r=0$, the potential becomes repulsive very quickly, and this is consistent with the expected short distance behavior we will see in next subsection.

If we continue past toward small $r$ beyond the region of validity (set by the integer $p$ ), the potential actually turns attractive again very sharply. However this is an artifact of cutting off the series at finite number of KK modes, and should not be taken seriously. Indeed, this unphysical turnaround can be seen to occur right below $1 / 3 M_{K K}$ where we expect the $p=10$ formula to become untrustworthy, at least for arbitrarily large $\lambda$. For finite $\lambda$, however, it turns out that there is a very simple remedy of this problem. The unphysical turnaround turns out to be a combined effect of the truncation and certain finite $\lambda$ correction that we ignored in section 4. By choosing an optimal value of $p$ in accordance with $\lambda$, one can easily restore physical sensible short-distance behavior as we explain in next subsection.

\subsection{Coulomb Repulsion at Short Distance and Finite $\lambda$ Cor- rections to the Large $N_{c}$ Potential}

When the distance between the pair of nucleon is much smaller than $1 / M_{K K}$ and comparable to $1 / \sqrt{\lambda} M_{K K}$, the above expressions must be summed over all mesons. When $\lambda$ is sufficiently large, however, it is clear where the leading contribution comes 
from. The holographic picture of the solitonic baryon involves an instanton soliton with a unit Pontryagin number dressed with Abelian electric charge. When the net soliton configuration is smaller than the curvature scale of the background holographic geometry, $1 / M_{K K}$, the instanton part of the soliton will behave like that of ordinary instanton on $R^{4}$ with scale invariance.

This implies that the leading potential energy beyond the rest masses of the two cores should come from the five-dimensional electrostatic energy associated with the Abelian electric charge. Roughly each soliton has $N_{c}$ unit of electric charges and the five dimensional electric coupling scales as $1 / \sqrt{\lambda N_{c}}$, and this gives repulsive potential

$$
\sim \frac{N_{c}}{\lambda} \frac{1}{M_{K K} r^{2}}
$$

Details of this potential are, however, more complicated. The electric charge density is basically the same as the Pontryagin density, so the precise form of the two-instanton solution enters the potential. In particular the relative spatial/gauge orientation of the two-instanton configuration must enter the potential, predicting a particular spin/isospin-dependence.

Clearly, the precise and quantitative structure of the short-distance potential cannot be captured by the our one-boson exchange potential since the underlying formulation for the latter ignores the core shape of the soliton other than its spin/isospin structures, whereas in the short-distance $\sim 1 / \sqrt{\lambda} M_{K K}$ the potential energy is of order $N_{c}$ and is comparable to the electric part of the soliton energy. In order to compute the precise structure of this short distance behavior, one should at least start from the full two-instanton solution, available in the literature either via ADHM construction or in the form of Jackiw-Nohl-Rebbi (JNR) ansatz [24]\#10 Unfortunately, however, this approach is difficult to extend beyond very short distance, since the analog of AHDM or JNR is not available in a curved background.

Independent of this, as a self-consistency check, we wish to understand how the sum over the KK tower of mesons end up producing $1 / r^{2}$ behavior at short distance. The leading short-distance power from individual meson exchange is $1 / r^{3}$. Since KK modes sum over such powers (after taking into account the coefficients carefully) cannot make a $1 / r^{2}$ form, somehow $1 / r^{3}$ terms must cancel in the full summation over mesons. For instance, pions contributes $\simeq 71 N_{c} / 4 \pi \lambda$ to the coefficient, whereas the $\rho$ and the first $a$ meson contribute $\simeq-80 N_{c} / 4 \pi \lambda$ and $\simeq 57 N_{c} / 4 \pi \lambda$, respectively. Continuing this fashion, one can see that the pion contribution is gradually eaten away by the alternating contributions from the pairs $\left(\rho^{(k)}, a^{(k)}\right)$. However, the sum up to $p=10$ can be seen to weaken $\sim 1 / r^{3}$ from the pion exchange only by a

\#10While our work was in progress, there appeared two related papers [25, 26] that share some common goal with our work. The latter in particular worked out a precise short-distance form of the potential using ADHM construction of two-instanton. 


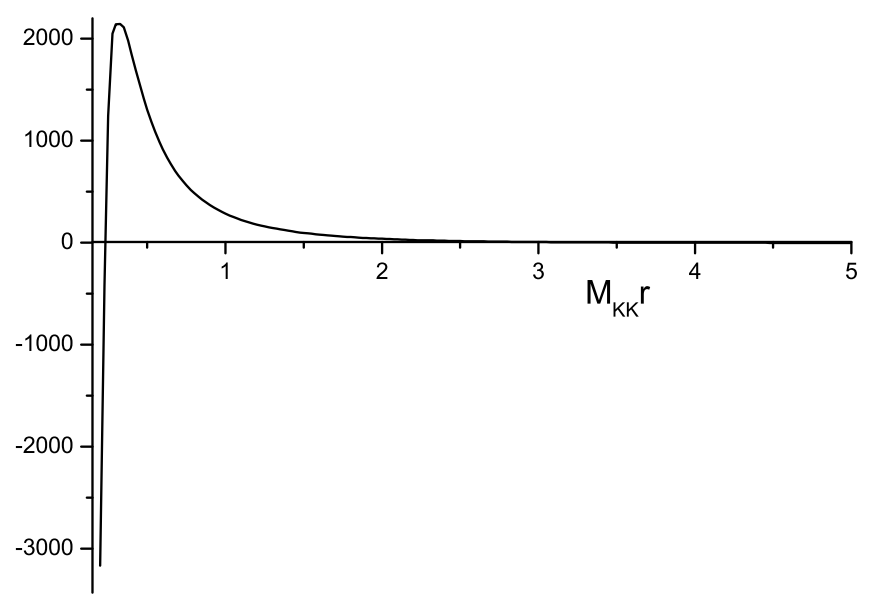

Figure 2: This plot shows the large $N_{c}$ potential $V_{(p)}$ at short distance where the naive large $\lambda N_{c}$ formula combined with the truncation becomes untrustworthy. Without the finite $\lambda$-correction, the truncated potential turns attractive again at a short distance of order $r M_{K K} \sim 3 / p$. The figure is for $p=10$.

factor of half, which is not enough for the anticipated cancellation. In numerical plot with $p=10$, shown in figure 2 , this manifests as an unphysical turnaround at $r M_{K K} \simeq 3 / 10$.

One reason behind this deficiency lies with the leading $\lambda N_{c}$ estimate we found in section 4 . While most of estimate there are safe in the large $N_{c}$ limit, the quantities $C_{n}^{ \pm}$are actually correct only up to $\sim 1 / \lambda$ corrections. This comes about because

$$
\frac{2 \pi^{2} \rho_{\text {baryon }}^{2}}{3 e(w)^{2}} \simeq \frac{\rho_{\text {baryon }}^{2}}{6} \times m_{\mathcal{B}}(w) \times\left(1-\frac{\sqrt{2 \cdot 3^{5} \cdot \pi^{2} / 5}}{\lambda}+O\left(\lambda^{-2}\right)\right)
$$

implying that our numbers for $g_{a^{(k)} \mathcal{N N}}$ were overestimated and we must adjust

$$
g_{a^{(k)} \mathcal{N N}} \rightarrow g_{a^{(k)} \mathcal{N N}} \times\left(1-\frac{\sqrt{2 \cdot 3^{5} \cdot \pi^{2} / 5}+}{\lambda}+O\left(\lambda^{-2}\right)\right)
$$

if we wish to understand finite $\lambda$ cases, regardless of $N_{c} \gg 1$.

Let us note that the smallest distance for which we can trust the truncation up to the $p$-th pair is around $r M_{K K} \sim 3 / p$. Comparing this distance against the solitonic baryon size, below which the effective theory we used does not make much sense to begin with, we find that such a truncated potential should be a sensible approximation 


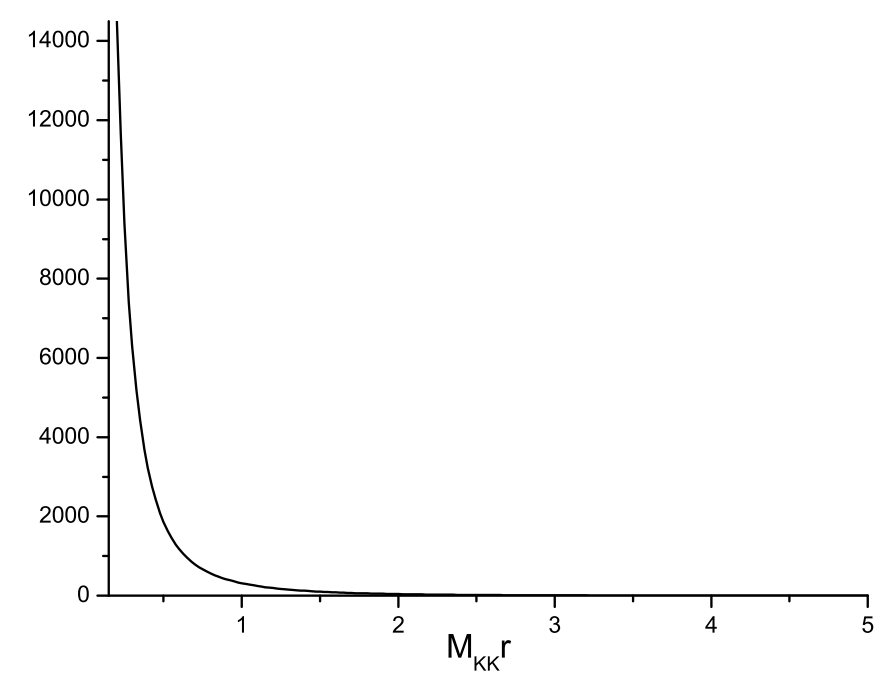

Figure 3: This plot shows the large $N_{c}$ potential $V_{(p=10)}$ now with $1 / \lambda$-corrected axial vector couplings at $\lambda=1100$. The unphysical turnaround at $\sim 0.3 M_{K K}$ disappeared completely, allowing a smooth transition to the short distance $1 / r^{2}$ repulsive core.

if we choose $p \sim \sqrt{\lambda / 10}$. For larger $p$, the idea of point-like nucleon fails as far as interaction with heavier mesons are concerned, while for smaller $p$ the potential $V_{(p)}$ fails at distances far larger than the individual baryon size.

For $\lambda \simeq 1000$, one may thus hope that the choice $p=10$ should be an optimal one. As we saw above, however, the naive formula for the potential exhibits that the potential begins to fails dramatically at $r M_{K K}=3 / p$, by turning strongly attractive again. Although there is no strong inconsistency with this (since the potential failed where it is expected to fail), it looks a little suspicious. The point is that although $\lambda \simeq 1000$ seems large, the correction (5.21) to the axial vector couplings represents roughly more than $2 \%$ reduction and cannot be neglected. What one should do is to correct $g_{a^{(k)} \mathcal{N N}}$ as in (5.21) and reconstruct the potential. Indeed, the numerical estimate shows an almost complete cancellation of short distance $1 / r^{3}$ when we take $p=10$ for the case of $\lambda=1100$. Figure 3 shows the corrected potential in this case, where the unphysical turnaround at $r M_{K K} \sim 3 / 10$ disappeared. In the case of $\lambda \sim 1000$, at least, the minimal choice for truncation, $p=10$, was also effective. Once this leaves behind $1 / r^{2}$ terms as the leading short-distance behaviors, the appearance of $1 / r^{2}$ from the latter via KK mode sum, where the vectors and the axial vectors contributes with the alternating sign, follows easily.

For larger $\lambda$, the reduction of $g_{a^{(k)} \mathcal{N N}}$ would be smaller, but at the same time we 
must sum over more mesons in order to make the potential trustworthy down to the distance comparable to the soliton core size. One may speculate that the optimal choice is again to sum up to $p \sim \sqrt{\lambda / 10}$. However, the couplings for large $k$ are inherently ambiguous since it depends more and more sensitively to, for example, the precise functional form of the coefficient of $\overline{\mathcal{B}} \mathcal{F} \mathcal{B}$. This is because the necessary mesonic wavefunction $\psi_{(n)}$ 's are more and more widespread, which also makes the couplings prone to systematic errors from how the numerical estimate is cut-off far away from $w=0$.

\section{Nucleon-Nucleon Potential for Realistic $\lambda$ and $N_{c}$}

If one wishes to understand real QCD with $N_{c}=3$, one must consider a different regime. For instance, we neglected $V_{\eta^{\prime}}$ on account of the small ratio

$$
\frac{g_{\eta^{\prime} \mathcal{N N}}}{g_{\pi \mathcal{N N}}} \sim \sqrt{\frac{3^{7} \cdot 5 \cdot \pi^{2}}{2^{3}}} \frac{1}{\lambda N_{c}} \ll 1 \quad \text { when } \lambda N_{c} \gg 1
$$

in the holographic limit. Yet, if we consider $N_{c}=3$ and $\lambda \simeq 17$ (determined by measured values of $f_{\pi}$ ), we find the ratio to be about 2 and is hardly ignorable. The estimates here themselves are no longer reliable since we used large $\lambda N_{c}$ limit, but this comparison clearly shows us that we cannot expect any small parameter. This is in fact a generic problem in going to the realistic limit.

In computing Feynman diagrams and extracting nonrelativistic potential, another small parameter is $\mathbf{p} / m_{\mathcal{N}}$ where $\mathbf{p}$ is the spatial momentum of the meson being exchanged. However, when translated to real space, this ratio can show up either as $m / m_{\mathcal{N}}$ or as $1 / r m_{\mathcal{N}}$, which is problematic when the meson mass $m$ exceeds the nucleon mass. Thus, contribution from exchange of heavy mesons cannot be included reliably, forcing us to cut down to pions, $\eta^{\prime}, \rho$, and $\omega$. Thanks to the universal suppression $\sim e^{-m r}$ for heavy meson processes, this is a good approximation as long as we are interested in distances strictly larger that $1 / M_{K K}$.

The relevant Nucleon-Nucleon potential is then

$$
V=V_{\pi}+V_{\eta^{\prime}}+V_{\rho^{(1)}}+V_{\omega^{(1)}}
$$

where individual term must be computed as a series expansion of $m / m_{\mathcal{N}}$. Actually, the exchange of vector mesons generates a correction to the kinetic term as well, the two-body Hamiltonian for a pair of nucleons contains the relative part of the Hamiltonian,

$$
H=-\left(\frac{1}{m_{\mathcal{N}}}+\Delta\right) \nabla^{2}+V
$$


with

$$
\Delta=\frac{3 m_{(1)}}{16 \pi}\left(g_{\omega^{(1)} \mathcal{N \mathcal { N }}}^{2}+g_{\rho^{(1)} \mathcal{N} \mathcal{N}^{2}}^{2} \cdot \vec{\tau}_{2}\right)\left(\frac{m_{(1)}}{m_{\mathcal{N}}}\right)^{2} y_{0}\left(m_{(1)} r\right),
$$

where $m_{(1)} \equiv m_{\omega^{(1)}}=m_{\rho^{(1)}}$. In the attractive channel, $\vec{\tau}_{1} \cdot \vec{\tau}_{2}=-3$, as we will see later, $\left(g_{\omega^{(1)} \mathcal{N N}} / g_{\omega^{(1)} \mathcal{N N}}\right)^{2} \simeq 14$, so the effective reduced mass of this two body system becomes smaller as the distance becomes small.

Let us turn to the potential. $V_{\pi}$ was already given in Eq. (5.11), while others can be inferred from Ref. [12]. The contribution from the trace part is essentially the same as the massive pion case (5.9) except the $S U(2)$ generators $\vec{\tau}_{1} \cdot \vec{\tau}_{2}$ are absent

$$
V_{\eta^{\prime}}=\frac{1}{4 \pi}\left(\frac{g_{\eta^{\prime} \mathcal{N N}}}{2 m_{\mathcal{N}}} M_{K K}\right)^{2} \frac{m_{\eta^{\prime}}^{2}}{M_{K K}^{2}} \frac{m_{\eta^{\prime}}}{3}\left[y_{0}\left(m_{\eta^{\prime}} r\right) \vec{\sigma}_{1} \cdot \vec{\sigma}_{2}+y_{2}\left(m_{\eta^{\prime}} r\right) S_{12}\right] .
$$

The mass of $\eta^{\prime}$ is generated by the $U(1)$ axial anomaly, and was computed by Sakai and Sugimoto,

$$
m_{\eta^{\prime}}=\frac{\lambda M_{K K}}{\sqrt{27 \pi^{2}}} \sqrt{\frac{N_{F}}{N_{c}}} .
$$

$V_{\rho^{(1)}}$ is considerably more involved than $V_{\rho^{(1)}}^{\text {holographic }}$ :

$$
\begin{aligned}
& V_{\rho^{(1)}}=\frac{m_{(1)}}{4 \pi}\left\{\left[g_{\rho^{(1)} \mathcal{N N}}^{2}\left(1-\frac{1}{4} \frac{m_{(1)}^{2}}{m_{\mathcal{N}}^{2}}\right)+g_{\rho^{(1)} \mathcal{N N}}\left(\frac{\tilde{g}_{\rho^{(1)} \mathcal{N N}}}{2 m_{\mathcal{N}}} M_{K K}\right) \frac{m_{(1)}}{m_{\mathcal{N}}} \frac{m_{(1)}}{M_{K K}}\right.\right. \\
& \left.+\frac{1}{4}\left(\frac{\tilde{g}_{\rho^{(1)} \mathcal{N N}}}{2 m_{\mathcal{N}}} M_{K K}\right)^{2}\left(\frac{m_{(1)}}{m_{\mathcal{N}}}\right)^{2}\left(\frac{m_{(1)}}{M_{K K}}\right)^{2}\right] y_{0}\left(m_{(1)} r\right) \\
& +\frac{1}{3} \frac{m_{(1)}^{2}}{M_{K K}^{2}}\left[\left(\frac{M_{K K}}{2 m_{\mathcal{N}}} g_{\rho^{(1)} \mathcal{N N}}+\frac{\tilde{g}_{\rho^{(1)} \mathcal{N N}}}{2 m_{\mathcal{N}}} M_{K K}\right)^{2}\right. \\
& \left.+\frac{1}{8}\left(\frac{\tilde{g}_{\rho^{(1)} \mathcal{N N}}}{2 m_{\mathcal{N}}} M_{K K}\right)^{2}\left(\frac{m_{(1)}}{M_{\mathcal{N}}}\right)^{2}\right]\left[2 y_{0}\left(m_{(k)} r\right) \vec{\sigma}_{1} \cdot \vec{\sigma}_{2}-y_{2}\left(m_{(k)} r\right) S_{12}(\hat{r})\right] \\
& -\left(\frac{m_{(1)}}{m_{\mathcal{N}}}\right)^{2}\left[\frac{3}{2} g_{\rho^{(1)} \mathcal{N N}}^{2}\right. \\
& \left.+2 g_{\rho^{(1)} \mathcal{N N}} \tilde{g}_{\rho^{(1)} \mathcal{N N}}+\frac{3}{2}\left(\frac{\tilde{g}_{\rho^{(1)} \mathcal{N N}}}{2 m_{\mathcal{N}}} M_{K K}\right)^{2}\left(\frac{m_{(1)}}{M_{K K}}\right)^{2}\right] \frac{y_{1}\left(m_{(1)} r\right)}{m_{(1)} r} \vec{L} \cdot \vec{S} \\
& +\left(\frac{m_{(1)}}{m_{\mathcal{N}}}\right)^{4}\left[\frac{1}{16} g_{\rho^{(1)} \mathcal{N N}}^{2}\right. \\
& \left.\left.+\frac{1}{2} g_{\rho^{(1)} \mathcal{N N}} \tilde{g}_{\rho^{(1)} \mathcal{N N}}+\frac{1}{2} \tilde{g}_{\rho^{(1)} \mathcal{N N}}^{2}\right] \frac{y_{2}\left(m_{(1)} r\right)}{m_{(1)}^{2} r^{2}} Q_{12}\right\} \times \vec{\tau}_{1} \cdot \vec{\tau}_{2},
\end{aligned}
$$


with

$$
\begin{aligned}
& \vec{S}=\frac{1}{2}\left(\vec{\sigma}_{1}+\vec{\sigma}_{2}\right), \\
& Q_{12}=\frac{1}{2}\left(\left(\vec{\sigma}_{1} \cdot \vec{L}\right)\left(\vec{\sigma}_{2} \cdot \vec{L}\right)+\left(\vec{\sigma}_{2} \cdot \vec{L}\right)\left(\vec{\sigma}_{1} \cdot \vec{L}\right)\right),
\end{aligned}
$$

and the spatial angular momentum $\vec{L}$. Finally, $V_{\omega^{(1)}}$ is essentially of the same form as $V_{\rho^{(1)}}$, except that $\tilde{g}_{\omega^{(1)} \mathcal{N N}}=0$ and $\vec{\tau}_{1} \cdot \vec{\tau}_{2}$ is absent,

$$
\begin{aligned}
V_{\omega(1)}= & \frac{m_{(1)}}{4 \pi} g_{\omega(1) \mathcal{N N}}^{2}\left\{\left(1-\frac{1}{4} \frac{m_{(1)}^{2}}{m_{\mathcal{N}}^{2}}\right) y_{0}\left(m_{(1)} r\right)\right. \\
& +\frac{1}{12}\left(\frac{m_{(1)}}{m_{\mathcal{N}}}\right)^{2}\left[2 y_{0}\left(m_{(1)} r\right) \vec{\sigma}_{1} \cdot \vec{\sigma}_{2}-y_{2}\left(m_{(1)} r\right) S_{12}(\hat{r})\right] \\
& \left.-\frac{3}{2}\left(\frac{m_{(1)}}{m_{\mathcal{N}}}\right)^{2} \frac{y_{1}\left(m_{(1)} r\right)}{m_{(1)} r} \vec{L} \cdot \vec{S}+\frac{1}{16}\left(\frac{m_{(1)}}{m_{\mathcal{N}}}\right)^{4} \frac{y_{2}\left(m_{(1)} r\right)}{m_{(1)}^{2} r^{2}} Q_{12}\right\} .
\end{aligned}
$$

These are the complete expressions up to the quartic order in terms of spatial momenta of individual nucleons.

For $N_{c}=3$ and $\lambda \simeq 17$, we found the following numbers that determine the couplings here,

$$
4 C_{0}^{+} \simeq 0.697, \quad 2 A_{0}^{+} \simeq 0.136, \quad A_{1}^{+} \simeq 5.93,2 B_{1}^{+} \simeq \frac{7.04}{M_{K K}}, 2 C_{1}^{+} \simeq-1.22
$$

and

$$
f_{\pi} \simeq 0.0975 M_{K K}, \quad m_{(1)} \equiv m_{\rho^{(1)}}=m_{\omega^{(1)}} \simeq 0.818 M_{K K}, \quad m_{\eta^{\prime}} \simeq 0.85 M_{K K} .
$$

The mass $m_{\mathcal{N}}$ has an inherent ambiguity since it would be additively renormalized by massive excitations around the soliton. Our definition of the nucleon mass kept only one such massive mode, namely the position along $w$-direction, and according to this prescription, we find

$$
m_{\mathcal{N}} \simeq 1.93 M_{K K}
$$

Unfortunately, the scale of $M_{K K}$ that fits the physical nucleon mass is about $500 \mathrm{MeV}$, as opposed to the one needed to fit the physical $\rho$ meson mass at $\sim 940 \mathrm{MeV}$. This discrepancy between the mesonic and the baryonic scales was previously observed both in the D4-D8 modelin a slightly different comparison [5] and also in the so-called bottom-up approach [28], and appears unavoidable in the gravity approximation to the bulk side. We will proceed with these numbers, nevertheless. The 


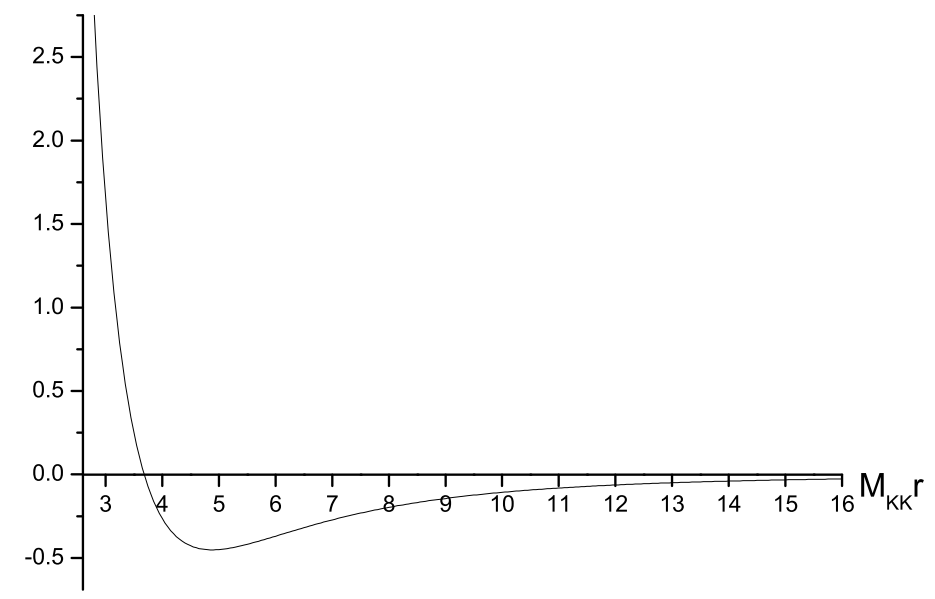

Figure 4: A plot of the nucleon-nucleon potential, with $N_{c} \lambda=50$ and $N_{c}=3$. We again drew the potential for the isospin singlet, $\sigma$-spin triplet, and $S_{12}=2$ eigensector, although for this finite $\lambda N_{c}$ case we should expect different $S_{12}$ eigensectors to mix in. This plot is only for the purpose of illustrating the general trend. The horizontal axis is $r M_{K K}$, while the vertical potential energy is in unit of $M_{K K} / 4 \pi$.

couplings that enter the above potential are\#11

$$
\begin{aligned}
\frac{g_{\pi \mathcal{N N}}}{2 m_{\mathcal{N}}} M_{K K} & =\frac{4 C_{0}^{+}+2 A_{0}^{+}}{2 f_{\pi}} M_{K K} \simeq 4.27, \\
\frac{g_{\eta^{\prime} \mathcal{N N}}}{2 m_{\mathcal{N}}} M_{K K} & =\frac{2 A_{0}^{+} \cdot N_{c}}{2 f_{\pi}} M_{K K} \simeq 4.18, \\
g_{\rho^{(1)} \mathcal{N N}} & =\frac{A_{1}^{+}+2 C_{1}^{+}}{2} \simeq 2.36, \\
g_{\omega}^{(1) \mathcal{N N}} & =\frac{A_{1}^{+} \cdot N_{c}}{2} \simeq 8.90 \\
\frac{\tilde{g}_{\rho^{(1)} \mathcal{N N}}}{2 m_{\mathcal{N}}} M_{K K} & =\frac{2 B_{1}^{+} \cdot M_{K K}}{2} \simeq 7.04 .
\end{aligned}
$$

Detailed study of this case will be reported elsewhere.

\#11 It has been observed previously that next subleading correction of some of the operators may involve the simple shift $N_{c} \rightarrow N_{c}+2$ in the leading expressions. This, for example, allows a very good match of $g_{\pi \mathcal{N N}}$ with experiment. The origin of this shift, originally suggested by the constituent quark models, is not clear from this approach. Here, we chose not to implement this shift but readers should be aware that terms from $\overline{\mathcal{B}} F \mathcal{B}$ may be affected, leading to quantitatively different numbers. 


\section{Holographic Deuteron: Large $N_{c}$ Results}

In this final section, we explore some basic aspects of deuteron physics with the NN potential

$$
V_{\pi}^{\text {holographic }}+\sum_{k=1}^{10}\left(V_{\rho^{(k)}}^{\text {holographic }}+V_{\omega^{(k)}}^{\text {holographic }}+V_{a^{(k)}}^{\text {holographic }}\right)
$$

in the large $\lambda$ and $N_{c}$ limit. To distinguish this from physical deuteron, we refer to them as holographic deuterons.

For a bound state, we need to focus on the long distance attractive channel. The large $N_{c}$ potential has a simple spin and flavor structure as

$$
V^{\text {holographic }}=V_{C}+\left(V_{T}^{\sigma} \vec{\sigma}_{1} \cdot \vec{\sigma}_{2}+V_{T}^{S} S_{12}\right) \vec{\tau}_{1} \cdot \vec{\tau}_{2}
$$

The massless pion exchange, the dominant contribution in long distance, contributes only to $V_{T}^{S}$ and positively, so an attractive channel requires $S_{12} \vec{\tau}_{1} \cdot \vec{\tau}_{2}<0$. Using the fact that $S_{12}$ acting on $\sigma$-spin singlet vanishes identically, and that the nucleons are fermions, this forces the isospin singlet $\left(\vec{\tau}_{1} \cdot \vec{\tau}_{2}=-3\right)$ and the $\sigma$-triplet $\left(\vec{\sigma}_{1} \cdot \vec{\sigma}_{2}=1\right)$ channel with even spatial angular momentum. The lowest total angular momentum possible is then $J=1$, and the positive $S_{12}$ eigensector has the following spatial angular momentum mix as

$$
\frac{|L=1\rangle+\sqrt{2}|L=0\rangle}{\sqrt{3}} .
$$

In this eigensector, $S_{12}=2$. The figure 1 is the plot of the potential in this sector, whose classical minimum occurs at

$$
r_{\min } \simeq \frac{5.53}{M_{K K}}, \quad V\left(r_{\min }\right) \simeq-0.0944 M_{K K} \frac{N_{c}}{\lambda} .
$$

Note that the binding potential is very shallow. Recall that in the large $\lambda N_{c}$ limit, $M_{K K}$ is most conveniently determined by the vector meson scale to be around $0.94 \mathrm{GeV}$. Among various scales that enter the baryon energy, we have the hierarchies,

$$
m_{\mathcal{N}} \sim m_{B}^{\text {classical }} \sim \lambda N_{c} \gg E_{\text {Coulomb }} \sim N_{c} \gg\left|V\left(r_{\text {min }}\right)\right| \sim \frac{N_{c}}{\lambda} .
$$

The middle measures the energy related to the classical deformation of the individual baryon away from the self-dual soliton, while the last measures the binding energy of the nuclei.

The fact that the nuclei biding energy is small, which is also borne out in real world \#12 is interesting from the standpoint of the holographic QCD as well. The

$\overline{\# 12}$ The physical deuteron has a binding energy of $2.2 \mathrm{MeV}$ [29], which is about $0.12 \%$ of its rest mass. 
stringy picture of the baryon says that the individual baryon can be viewed as a D4 brane wrapped on the compact $S^{4}$ of the dual geometry [27, 1]. What we computed here is essentially the potential between two such objects separated along the noncompact $R^{3}$. The binding energy is positive but suppressed relative to the individual rest mass by $1 / \lambda^{2}$, indicating very weak interactions. In terms of the warped string scale, $\alpha_{\text {warped }}^{\prime}$, this power is equals $\left(\alpha_{\text {warped }}^{\prime} M_{K K}^{2}\right)^{2}$. Although the significance of this particular power is unclear to us, it does show that the two wrapped D4 branes are almost non-interacting at long distances. This seems to suggest that the object underlying baryons may remain close to its original BPS nature, despite the supersymmetry breaking background of scale $M_{K K}$ and high mass $\sim \lambda N_{c} M_{K K}$, which is well beyond the cut-off scale $M_{K K}$, and may eventually explain why such a high mass object is well-described by this D4-D8 holographic QCD.

\section{Concluding Remarks}

In this work, we computed the nucleon-nucleon potential in the D4-D8 holographic QCD, which is generated by exchange of five-dimensional flavor gauge field. In four dimensional picture, this amounts to exchange of massless pseudo-scalars and an infinite tower of spin one mesons. In the large $\lambda N_{c}$ limit, it is sensible to sum up to first $\sim \sqrt{\lambda / 10}$ vector and axial vector meson pairs, although one may choose to cut it shorter according to the shortest distance scale interested. This prescription also gives whereto glue the repulsive short distance regime to the more complicated intermediate and long distance regime. Some rudimentary aspects of deuteronic bound state is explored for large $N_{c}$ case. Consideration of deuteron for realistic QCD regime will be explored elsewhere.

We hope this work will provide a more practical starting point for exploration of how holographic QCD fares against experimental data, part of which comes from nucleon-nucleon scattering amplitudes. Admittedly, this would involved huge extrapolation to $N_{c}=3$ and $\lambda \simeq 17$, where the holographic approach is hardly justifiable by the first principle. But, in the absence of any other honest derivation of nucleonnucleon potential, our result should be at least tested against data. In this work, we did not attempt to analyze realistic QCD regime and concentrated mostly on large $N_{c}$ limit. We wish to come back later to the $N_{c}=3$ potential of section 6 , and explore its consequences.

Another important application of this work would be in understanding dense matter system, such as neutron stars, where the correct equation of state is of some importance. In dealing with such a dense system from the holographic QCD, baryon density itself were often treated as external input in the form of delta-function density in five-dimensions. We hope our nucleon-nucleon potential would allow a more refined approach. 


\section{Acknowledgments}

P.Y. is grateful to Lenny Susskind for a comment that motivated this work, and also Deog-Ki Hong, Shamit Kachru, Mannque Rho, and Ho-Ung Yee for discussions. He also thanks SITP of Stanford University for hospitality and generous support. Y.K. thanks Hyun-Chul Kim for useful comments. Y.K. acknowledges the Max Planck Society(MPG) and the Korea Ministry of Education, Science and Technology(MEST) for the support of the Independent Junior Research Group at the Asia Pacific Center for Theoretical Physics (APCTP). S.L. is supported in part by the KOSEF Grant R01-2006-000-10965-0 and the Korea Research Foundation Grant KRF-2007-331C00073. P.Y. is supported in part by the Science Research Center Program of KOSEF (CQUeST, R11-2005-021), the Korea Research Foundation (KRF-2007-314-C00052), and by the Stanford Institute for Theoretical Physics (SITP Quantum Gravity visitor fund).

\section{References}

[1] T. Sakai and S. Sugimoto, Prog. Theor. Phys. 113, 843 (2005) arXiv:hep-th/0412141;

Prog. Theor. Phys. 114, 1083 (2006) [arXiv:hep-th/0507073].

[2] C. Csaki, H. Ooguri, Y. Oz and J. Terning, JHEP 9901, 017 (1999) arXiv:hep-th/9806021.

[3] R. C. Brower, S. D. Mathur and C. I. Tan, Nucl. Phys. B 587, 249 (2000) arXiv:hep-th/0003115.

[4] D. K. Hong, M. Rho, H. U. Yee and P. Yi, Phys. Rev. D 76, 061901, (2007) arXiv:hep-th/0701276.

[5] H. Hata, T. Sakai, S. Sugimoto and S. Yamato, arXiv:hep-th/0701280].

[6] D. K. Hong, M. Rho, H. U. Yee and P. Yi, JHEP 0709, 063 (2007) arXiv:0705.2632 [hep-th]].

[7] H. Imai, A. Kobayashi, H. Otsu and S. Sawada, Prog. Theor. Phys. 82, 141 (1989).

[8] R. A. Leese, N. S. Manton and B. J. Schroers, Nucl. Phys. B 442 (1995) 228 arXiv:hep-ph/9502405.

[9] J.J. De Swart, P.M.M. Maessen, and T.A. Rijken, nucl-th/9405008. 
[10] M.M. Nagels, T.A. Rijken, and J.J. de Swart, Phys. Rev. D12, 744 (1975); D17, 768 (1978); R. Machleidt, K. Holinde, and C. Elster, Phys. Rept. 149, 1 (1987).

[11] J.W. Durso, G.E. Brown, and M. Saarela, Nucl. Phys. A430, 653 (1984).

[12] T. Ericson and W. Weise, Pions and Nuclei, Oxford 1988.

[13] E. Witten, Adv. Theor. Math. Phys. 2, 505 (1998) arXiv:hep-th/9803131.

[14] J. M. Maldacena, Adv. Theor. Math. Phys. 2, 231 (1998) arXiv:hep-th/9711200]; S. S. Gubser, I. R. Klebanov and A. M. Polyakov, Phys. Lett. B 428, 105 (1998) arXiv:hep-th/9802109]; E. Witten, Adv. Theor. Math. Phys. 2, 253 (1998) arXiv:hep-th/9802150.

[15] G. W. Gibbons and K. I. Maeda, Nucl. Phys. B 298 (1988) 741.

[16] T.H.R. Skyrme, Nucl. Phys. 31, (1962) 556.

[17] G. 't Hooft, Nucl. Phys. B 72 (1974) 461.

[18] M. F. Atiyah and N. S. Manton, Phys. Lett. B 222, (1989) 438.

[19] T. Fujiwara et al, Theor. Phys. 74, 128 (1985); U.-G. Meissner, N. Kaiser, A. Wirzba and W. Weise, Phys. Rev. Lett. 57, 1676 (1986); U.G. Meissner and I. Zahed, Phys. Rev. Lett. 56, 1035 (1986); K. Nawa, H. Suganuma and T. Kojo, Phys. Rev. D 75 (2007) 086003 [arXiv:hep-th/0612187].

[20] D. Finkelstein and J. Rubinstein, J. Math. Phys. 9, 1762 (1968).

[21] G.S. Adkins, C.R. Nappi and E. Witten, Nucl. Phys. B228, 552 (1983).

[22] J. Park and P. Yi, JHEP 0806 (2008) 011 [arXiv:0804.2926 [hep-th]].

[23] K. Hashimoto, T. Sakai and S. Sugimoto, Prog. Theor. Phys. 120 (2008) 1093 arXiv:0806.3122 [hep-th]].

[24] R. Jackiw, C. Nohl and C. Rebbi, Phys. Rev. D 15, (1977) 1642.

[25] K. Y. Kim and I. Zahed, arXiv:0901.0012 [hep-th].

[26] K. Hashimoto, T. Sakai and S. Sugimoto, arXiv:0901.4449 [hep-th].

[27] E. Witten, JHEP 9807, 006 (1998) arXiv:hep-th/9805112.

[28] D. K. Hong, T. Inami and H. U. Yee, Phys. Lett. B 646 (2007) 165 arXiv:hep-ph/0609270. 
[29] See for example, M. Garcon and J. W. Van Orden, Adv. Nucl. Phys. 26, 293 (2001) arXiv:nucl-th/0102049]. 